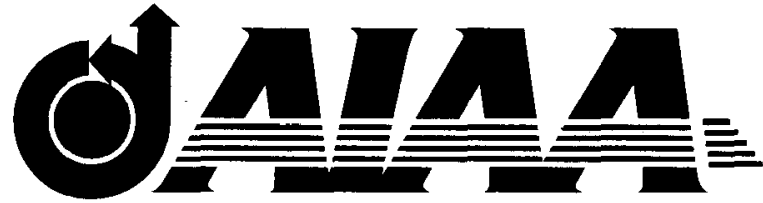

\section{AIAA 98-2668}

Shock-Wave/Boundary-Layer Interactions in Hypersonic Low Density Flows

James N. Moss

NASA Langley Research Center Hampton, VA 23681-0001

Joseph Olejniczak University of Minnesota Minneapolis, MN 55455

\section{7th AIAA/ASME Joint Thermophysics and Heat Transfer Conference June 15-18, 1998 / Albuquerque, NM}




\title{
SHOCK-WAVE/BOUNDARY-LAYER INTERACTIONS IN HYPERSONIC LOW DENSITY FLOWS
}

\author{
James N. Moss* \\ Mail Stop 408A \\ NASA Langley Research Center \\ Hampton, VA 23681-0001 \\ e-mail: j.n.moss@larc.nasa.gov \\ and \\ Joseph Olejniczak ${ }^{\dagger}$ \\ Department of Aerospace Engineering and Mechanics \\ Army High Performance Computing Research Center \\ University of Minnesota \\ Minneapolis, MN 55455
}

\begin{abstract}
$\underline{\text { Abstract }}$
Results of numerical simulations of Mach 10 air flow over a hollow cylinder-flare and a double-cone are presented where viscous effects are significant. The flow phenomena include shock-shock and shockboundary-layer interactions with accompanying flow separation, recirculation, and reattachment. The purpose of this study is to promote an understanding of the fundamental gas dynamics resulting from such complex interactions and to clarify the requirements for meaningful simulations of such flows when using the direct simulation Monte Carlo (DSMC) method. Particular emphasis is placed on the sensitivity of computed results to grid resolution. Comparisons of the DSMC results for the hollow cylinder-flare $\left(30^{\circ}\right)$ configuration are made with the results of experimental measurements conducted in the ONERA R5Ch wind tunnel for heating, pressure, and the extent of separation. Agreement between computations and measurements for various quantities is good except that for pressure. For the same flow conditions, the doublecone geometry $\left(25^{\circ}-65^{\circ}\right)$ produces much stronger interactions, and these interactions are investigated numerically using both DSMC and Navier-Stokes

\footnotetext{
*Aerospace Engineer, Aerothermodynamics Branch, Aeroand Gas-Dynamics Division, Fellow AIAA.

†Post-Doctoral Research Associate, Member AIAA
}

Copyright $\odot 1998$ by the American Institute of Aeronautics and Astronautics, Inc. No copyright is asserted in the United States under Title 17, U.S. Code. The U.S. Government has a royalty-free license to exercise all rights under the copyright claimed herein for governmental purposes. All other rights are reserved by the copyright owner.
\end{abstract}

codes. For the double-cone computations, a two orders of magnitude variation in free-stream density (with Reynolds numbers from 247 to 24,719 ) is investigated using both computational methods. For this range of flow conditions, the computational results are in qualitative agreement for the extent of separation with the DSMC method always predicting a smaller separation region. Results from the Navier-Stokes calculations suggest that the flow for the highest density double-cone case may be unsteady; however, the DSMC solution does not show evidence of unsteadiness.

\section{Nomenclature}

$\begin{array}{ll}\text { A } & \text { base area of cone, } \pi \mathrm{d}^{2} / 4 \\ \mathrm{C} & \text { Chapman-Rubesin constant } \\ \mathrm{C}_{\mathrm{f}} & \text { skin-friction coefficient, } 2 \tau / \rho_{\infty} \mathrm{V}_{\infty}^{2} \\ \mathrm{C}_{\mathrm{D}} & \text { drag coefficient, } 2 \mathrm{D} / \mathrm{\rho}_{\infty} \mathrm{V}_{\infty}^{2} \mathrm{~A} \\ \mathrm{C}_{\mathrm{D}, \mathrm{P}} & \text { drag coefficient due to pressure } \\ \mathrm{C}_{\mathrm{H}} & \text { heat transfer coefficient, } 2 \mathrm{q} / \rho_{\infty} \mathrm{V}_{\infty}^{3} \\ \mathrm{C}_{\mathrm{p}} & \text { pressure coefficient, } 2\left(\mathrm{p}_{\mathrm{w}}-\mathrm{p}_{\infty}\right) / \rho_{\infty} \mathrm{V}_{\infty}^{2} \\ \mathrm{C}_{\mathrm{p}}^{\prime} & \text { modified pressure coefficient, } 2 \mathrm{p}_{\mathrm{w}} / \rho_{\infty} \mathrm{V}_{\infty}^{2} \\ \mathrm{D} & \text { drag } \\ \mathrm{M} & \text { Mach number } \\ \text { n } & \text { number density }\end{array}$




\begin{tabular}{|c|c|}
\hline $\mathbf{p}$ & pressure \\
\hline q & heat transfer rate \\
\hline $\mathbf{R}$ & denotes point of reattachment \\
\hline $\operatorname{Re}_{\infty, \mathrm{d}}$ & $\begin{array}{l}\text { Reynolds number based on diameter, } \\
\rho_{\infty} V_{\infty} d / \mu_{\infty}\end{array}$ \\
\hline $\operatorname{Re}_{\infty, L}$ & $\begin{array}{l}\text { Reynolds number based on length, } \\
\rho_{\infty} \mathrm{V}_{\infty} \mathrm{L} / \mu_{\infty}\end{array}$ \\
\hline $\mathbf{s}$ & $\begin{array}{l}\text { distance along cone surface measured from } \\
\text { apex }\end{array}$ \\
\hline $\mathbf{S}$ & denotes point of separation \\
\hline $\mathrm{T}$ & temperature \\
\hline $\mathrm{T}_{\mathrm{w}}$ & wall temperature \\
\hline $\mathbf{u}$ & velocity in $\mathrm{x}$-direction \\
\hline V & speed \\
\hline$\overline{\mathrm{V}}$ & rarefaction parameter, $\mathrm{M}_{\infty} \sqrt{\mathrm{C} / \mathrm{Re}_{\infty}}$ \\
\hline $\mathbf{x}, \mathbf{y}$ & Cartesian coordinates \\
\hline$\mu$ & viscosity \\
\hline$\rho$ & density \\
\hline$\tau$ & shear stress \\
\hline $\bar{x}$ & $\begin{array}{l}\text { hypersonic viscous interaction parameter, } \\
\bar{X}=M_{\infty}^{2} \bar{V}\end{array}$ \\
\hline
\end{tabular}

Subscripts

C cone

d maximum diameter of double cone

L distance from cylinder leading edge to flare

$\mathrm{L}_{\mathrm{C}} \quad$ length of cone (see Fig. 7)

w wall

$\infty \quad$ free stream

\section{Introduction}

Shock-wave/boundary-layer interactions are a major concern in hypersonic flows because of the impact on vehicle stability and the potential for causing very high localized aerothermal loads in the interaction region. The physics of such flows is influenced by a large variety of factors ${ }^{1}$, some of which are geometry, Reynolds number, flow enthalpy, and boundary conditions. In the present study, the focus is on the interactions resulting from Mach 9.91 air flow over two configurations at low density conditions with Reynolds numbers of 25,000 or less. For these Reynolds numbers, both the separation and reattachment events occur under laminar conditions. Direct simulation Monte Carlo (DSMC) results are presented for a hollow cylinder with a $30^{\circ}$ flare and for of a double cone with cone half angles of $25^{\circ}$ and $65^{\circ}$.

Computations for the hollow cylinder-flare configuration are presented first, where the current DSMC results are an extension and refinement of the results given in Refs. 2 and 3. The present calculations provide more than a factor of four increase in the spatial resolution by using 4 subscells per cell in the simulation; collision partners are selected on a subcell basis while the macroscopic results are still presented on a cell basis. Details of the current results for flowfield structure and surface distributions are presented. Also, comparisons of the DSMC results for surface heating, pressure, and the extent of separation are made with the corresponding data obtained in the ONERA R5Ch hypersonic wind tunnel at Chalais-Meudon, France. Details of the ONERA experiments for the hollow cylinder-flare model are given in Refs. 4 and 5. A forthcoming report (Ref. 6) will provide a synthesis of the hollow cylinder-flare problem, which was one of the test problems at the First Europe-US High Speed Flow Field Database Workshop Part II, Naples, Italy in November 1997. Thirteen computational solutions were presented for this test case, which was selected for validation of Navier-Stokes solvers for cold, high-speed flow conditions with strong shock-wave/boundary-layer interactions in a fully laminar regime.

Results of recent numerical and experimental investigations (Refs. 7-9) have been reported on the shock interactions resulting from hypersonic flows over axisymmetric double-cone geometries. These investigations included both cold and high enthalpy flows where the emphasis was to identify the underlying gas dynamics of the interactions and the sensitivity to real gas effects. The high enthalpy experiments, conducted in the Caltech Free-Piston Shock Tunnel (T5), were specifically designed ${ }^{9}$ to be sensitive to vibration-dissociation coupling. The parameter space covered in these tests included variations in the second cone angle (half angles of $65^{\circ}$, $68^{\circ}$, and $70^{\circ}$ ) and the free-stream enthalpy (24.5 to $30.8 \mathrm{MJ} / \mathrm{kg}$ ). The flow conditions were Mach 6.5 to 8 nitrogen with a significant level of atomic nitrogen (about 0.2 mass fraction). The investigations showed that the Navier-Stokes computations did not reproduce the experimentally observed features of the separation 
zone using current thermo-chemical models, and that these complex flows could be a more sensitive discriminator for model validation than blunt-body flows. The cold hypersonic experiments were conducted in the Princeton University Mach 8 wind tunnel at free-stream Reynolds numbers (based on maximum cone diameter) of 370,000 and 610,000 . Two model configurations were tested at each of the two flow conditions-a $25^{\circ}-35^{\circ}$ double cone and a $25^{\circ}-50^{\circ}$ double cone. Common to both the low and high enthalpy test models is the size of the first cone angle $\left(25^{\circ}\right.$ half angle) and the fact that both cones are of equal length. Comparison between the experiments and computations (separation size and pressure distribution) is excellent for the Type VI shock interaction observed for the $25^{\circ}-35^{\circ}$ cone. However, for the Type $V$ shock interaction observed for the $25^{\circ}-50^{\circ}$ cone, the NavierStokes computations over-predicted the size of the separation region. Also for the $50^{\circ}$ second cone, the behavior of the experimental results (separation size decreasing with Reynolds number) may indicate that transition to turbulence is occurring due to the separation and/or reattachment shocks. These investigations (Refs. 7-9) highlight many of the issues associated with obtaining experimental and computational results that can be used for validating complex physical phenomena.

The objectives of the present DSMC double-cone computations are to identify the basic shock layer flow features where complex shock and shock-boundarylayer interactions are present in low Reynolds number flow and to investigate the spatial grid resolution required to achieve meaningful results. This is accomplished with the DSMC 2D/axisymmetric code of Bird $^{10-11}$ where the flow conditions are the same as those used in the hollow cylinder-flare study-Mach 9.91 air with a free-stream Reynolds number of 24,719. With respect to this flow condition, the free-stream density is decreased parametrically by two orders of magnitude to demonstrate the effects of rarefaction on the nature of the flow structure. In the absence of experimental results for the present low-density test cases, the DSMC results are compared with NavierStokes solutions obtained by the second author using the recently developed implicit Data-Parallel Line Relaxation (DPLR) method of Wright et al. ${ }^{12}$; the same Navier-Stokes solver is used in the investigations of Refs. 7-9. The geometry selected for investigation is a $25^{\circ}-65^{\circ}$ double cone with a $13.29 \mathrm{~cm}$ base diameter, the same size and configuration as one of the doublecone models used in the T5 series of tests.

\section{Numerical Methods}

The DSMC method provides a numerical capability that acknowledges the discrete nature of the gas and thereby provides a capability of simulating flows across the complete flow spectrum of continuum to free molecular. However, the computing requirements can become very demanding for multi-dimensional continuum applications. The DSMC code used in the current study is the general 2D/axisymmetric code of Bird $^{10-11}$. The molecular collisions are simulated using the variable hard sphere (VHS) molecular model. This model employs the simple hard sphere angular scattering law so that all directions are equally possible for post-collision velocity in the center-of-mass frame of reference. However, the collision cross section is a function of the relative energy in the collision. Energy exchange between kinetic and internal modes is controlled by the Larsen-Borgnakke statistical model ${ }^{13}$. For the present study, the simulations are performed using a nonreacting gas model consisting of two species, while considering energy exchange between translational, rotational, and vibrational modes. A rotational relaxation collision number of 5 was used for the calculations. The vibrational collision number was 50. The reference conditions for the VHS model were as follows: reference temperature $=300 \mathrm{~K}$, temperature exponent of the viscosity coefficient $=0.75$, and reference diameters for $\mathrm{O}_{2}$ and $\mathrm{N}_{2}$ were $3.96 \times 10^{-10} \mathrm{~m}$ and $4.07 \times 10^{-10} \mathrm{~m}$, respectively. The model surface is assumed to have a constant temperature of $293 \mathrm{~K}$. Full thermal accommodation and diffuse reflection are assumed for the gas-surface interactions.

For the Navier-Stokes computations, the axisymmetric equations are solved using a modified form of Steger-Warming flux vector splitting ${ }^{14}$, which gives second order spatial accuracy in the bodytangential direction. A no-slip velocity condition was applied at the body surface, and an isothermal wall temperature of $293 \mathrm{~K}$ was assumed for all cases. The implicit Data-Parallel Line Relaxation (DPLR) method $^{12}$ was used to obtain steady-state solutions. Due to the complex nature of these shock interaction flows, a large number of grid points is required to ensure a grid converged solution. In this paper, all solutions were obtained using a grid size of $512 \times 512$ points. A grid sensitivity study showed that a solution obtained on a $256 \times 256$ point grid was essentially identical to the solution obtained on a $512 \times 512$ point grid. However, it was decided to use the $512 \times 512$ point grid 
to ensure grid converged solutions for all the cases considered. The solutions were generated using a Sutherland viscosity law and a Prandtl number of 0.71 . Grid adaption was limited to fitting the outer grid boundary to the shock wave, stretching at the wall, and stretching around the corner in the body tangential direction.

\section{A. Hollow Cylinder Flare Results}

The hollow cylinder-flare test case considered here was formulated as one of the test problems concerning shock-wave/boundary-layer interactions in the AGARD WG18 activity (described in Ref. 1) for the validation of Navier-Stokes solvers for cold high-speed flows where the interactions produce large separated regions under laminar conditions. This test case has generated, and continues to generate, interest for code validation as evidenced by participation in the AGARD activity ${ }^{15}$ and a more recent workshop that will be summarized in Ref. 6.

A motivation for investigating this test case with DSMC is more in the spirit of establishing the level of grid resolution one must use to achieve accurate computational results for problems with complex interactions. Consequently, this test case is attractive for testing DSMC results since it is an axisymmetric problem that reduces the computational burden and surface measurements have been reported ${ }^{4-5}$. The present computational investigation is a continuation of the first author's ${ }^{2-3}$ computations for this test case with significant additional grid refinement. Before discussing the results, a brief description of the the model configuration, nominal test conditions, and computational grid is presented.

\section{Model Configuration and Test Conditions}

An experimental test program has been conducted ${ }^{4-5}$ by the Office National d'Etudes et de Recherches Aerospatials (ONERA) to investigate shock-boundarylayer interactions induced by a hollow cylinder-flare configuration. Figure 1 presents a schematic of the axisymmetric test model along with dimensions given in $\mathrm{mm}$. The hollow cylinder has a sharp leading edge with a bevel angle of $15^{\circ}$ and is aligned parallel to the oncoming flow. The compression flare is inclined $30^{\circ}$ to the cylinder and is terminated by a hollow cylindrical section. The total length of the model is $0.17 \mathrm{~m}$. The experiments were conducted in the ONERA R5Ch wind tunnel using a contoured nozzle that provides a uniform free-stream environment $0.2 \mathrm{~m}$ in diameter. The nominal stagnation conditions for the tests were 2.5 bars and $1050 \mathrm{~K}$ which provides upstream air flow properties of

$$
\begin{aligned}
& \mathrm{M}_{\infty}=9.91 \\
& \mathrm{~T}_{\infty}=51 \mathrm{~K} \\
& \mathrm{p}_{\infty}=6.3 \mathrm{~Pa} \\
& \operatorname{Re}_{\infty} / \mathrm{m}=1.86 \times 10^{5}
\end{aligned}
$$

where the viscosity $\left(3.290 \times 10^{-6} \mathrm{~Pa} \bullet \mathrm{s}\right)$ is that given by the Sutherland expression. The Reynolds number based on the model length from the leading edge to the flare $(0.1017 \mathrm{~m})$ is equal to $\operatorname{Re}_{\infty, \mathrm{L}}=18,916$. The available experimental data ${ }^{4-5}$ are surface pressure, heat transfer rates, and oil-flow data along with flow-field visualization by the Electron Beam Fluorescence (EBF) technique.

As discussed in some detail in Ref. 2, much of the flow over the cylinder is in the strong viscous interaction region as indicated by the $\bar{V}$ parameter $\left(\overline{\mathrm{V}}=\mathrm{M}_{\infty} \sqrt{\mathrm{C} / \mathrm{Re}_{\infty}}\right)$. Within the strong interaction region, the development of the viscous and inviscid flow are strongly coupled due to the rapid boundarylayer growth. A $\bar{V}$ value of approximately 0.15 is often quoted as the boundary between strong interaction and merged layer regions. For the current problem, a $\bar{V}=0.15$ corresponds to an $x$-location of $0.021 \mathrm{~m}$. At the cylinder-flare juncture, the value of $\bar{V}$, based on free-stream conditions is 0.068 or $\bar{X}=6.7$ $\left(\bar{X}=\overline{\mathrm{V}} \mathrm{M}_{\infty}^{2}\right.$ ). Close to the leading edge, the rarefaction effects are more significant as the nonequilibrium effects are manifested in terms of both the shock structure (the thin Rankine-Hugoniot shock structure breaks down-see Ref. 16, Fig. 12) and surface slip and temperature jump effects. ${ }^{2}$

\section{Computational Flow Domain and Grid}

The computational domain is made large enough so that the upstream and side boundaries can be specified as free-stream conditions. The flow at the down-stream outflow boundaries is supersonic and vacuum conditions are specified. Figure 2 shows the physical extent of the computational domain for the present simulations, which is subdivided into 4 regions with each region subdivided into computational cells. A significant difference in the current calculations and that of Refs. $2-3$ is that each cell is further subdivided into subcells. The number of subdivisions is arbitrary, 
but $4(2 \times 2)$ subcells/cell were used where the cells are subdivided in each direction. In the current DSMC code, the collision partners are selected from the same subcell; consequently, the flow resolution is much higher than the cell resolution (however, the macroscopic properties are extracted from averages within the cell).

Included in Fig. 2 are details for three different grid resolutions considered in the present investigation. Information concerning the number of cells in the axial and radial directions and the time steps for each region is listed for each of the three grids.

For data concerning noncontinuum surface results for leading edge regions, the DSMC results presented in Ref. 2 give a more detailed description where additional resolution of this region of the flow was employed. This difference in resolution was accomplished by using a small region that extended downstream of the leading edge and using smaller cell spacings in the axial direction than the current study. The effect of the additional resolution in the leading edge region was confined to the leading edge region. The present simulations concentrate the resources on the separation and reattachment regions of the flow domain.

\section{Computational Results}

Effect of Grid Resolution. A check on the effect of using 4 subcells/cell was first made by repeating the calculations made with the fine grid $(38,925$ cells) computations presented in Ref. 3. Calculations with and without subcells were made, and the results with subcells showed improved agreement with the experimental surface measurements along the flare. Subsequently, results for the three grids described in Fig. 2 were generated. The sensitivity of the surface results to grid resolution for skin friction, heat transfer rate, and surface pressure is demonstrated for these three grids in Fig. 3. The skin friction distributions (Fig. 3a) show that the extent of the separation region is quite sensitive to the grid-a much smaller separation region is obtained with the coarse grid. Changes in the location of separation and reattachment have a significant impact on the surface results downstream of separation. Tabulated information on the location of separation, reattachment, and the extent of separation is included in Table 1.

Note that the end of the flare is located at an $x / L$ location of 1.426, where the flow can expand onto the cylindrical extension. As will be demonstrated later, the peak pressure realized on the flare is being influenced by the corner expansion, which would not be the case if the flare were longer, and, to some extent, this complicates the interpretation of the grid effect on the local conditions along the flare. For example, to what extent are the larger peak pressures and heat transfer rates on the flare due to the local grid and to what extent are they due to the forward movement of reattachment with potentially less influence of the outer corner expansion on the flare peak pressure? Neverless, the effect of gridding is pervasive and very significant for these flows.

Note that the grid adjustments among the three grids is very selective. For the fine and intermediate grids, the cells and time steps are identical for regions 1 and 4. For the fine grid, attention is placed on the region where separation occurs (region 2) and, particularly, along the flare (region 3) where the higher density flow conditions and reattachment occurs. In region 3 , there are 45,000 cells for the fine grid, 14,400 for the intermediate grid, and only 3,600 for the coarse grid. Yet the differences in the computed surface quantities are rather small for the fine and intermediate grids. For the coarse grid, reductions in the number of cells are made for all regions with respect to the intermediate grid. For region 1, the reduction was 40 percent (only in the radial direction), and this had no precipitable effect on the surface results within region 1.

Comparison With Surface Measurements. Figure 4 presents a comparison of the current fine grid results with measurements made in the ONERA R5Ch tunnel. Figure $4 \mathrm{a}$ shows a comparison of the calculated skin friction coefficient distribution with the separation and reattachment locations inferred from oil flow measurements ${ }^{4-5}$. Information concerning the extent of the separation region is fundamental to the heating and pressure results, particularly on this relatively short flare.

Note that new oil flow experiments ${ }^{5}$ have been performed (with changes in the oil and marker composition) that provide improved definition of the attachment line, and the experimental results shown in Fig. $4 \mathrm{a}$ and Table 1 are the new results which give a 7 percent larger $(x / L)$ separation extent than the data reported in Ref. 4. As seen from Fig. 4 and Table 1, the overall agreement between calculation and measurement is good. The separation location is the same. The calculated reattachment location occurs, however, somewhat forward of the experimental value, and the extent of the calculated separation $(\Delta x / L)$ is 98 percent of the measurement. Note that the DSMC results presented in Ref. 3 with a grid consisting of 
38,925 cells and no subcells gave a separation extent that was much smaller ( 83 percent) than the current experimental value. Yet, the current intermediate grid consisting of almost the same number of cells $(39,400)$ and 4 subcells/cell predicts a separation extent that is 93 percent of the measurement.

These computational findings clearly demonstrate the requirement of very high spatial resolution for such problems. Furthermore, a significant increase in computational efficiency could potentially be realized if the number of simulated molecules and the spatial resolution (both defined and adapted) were better adaped to the local flow conditions.

The heating rate comparisons are given in Fig. 4b, and the overall agreement is very good. The distributions are characteristic of those for laminar flows in that the heating shows an initial decrease at the location of separation ( $\mathrm{x} / \mathrm{L}=0.76$ ), a cusp-like behavior at the juncture, and a rapid increase in heating rate along the flare. For this problem, the maximum heating rate occurs downstream of reattachment, which occurs just before the expansion onto the cylindrical extension.

As described by Chanetz et al. ${ }^{5}$, the heat transfer model was made of steel with a ceramic insert on which 24 platinum films were installed for the measurements from which the heating rates are inferred. Two locations of some discrepancy are noted. One is at the onset of the measurements $(x / L=0.2)$, where the flow is making the transition from a merged layer regime to one of strong interaction (based on $\bar{V}$ value of 0.15 ). The computational results are higher than the measured values at this location. The other location occurs after the cylinder-flare juncture, where the measured values are higher than the calculated results.

Surface pressure comparisons are shown in Fig. 4c, where the data is expressed in terms of the pressure coefficient. The measurements ${ }^{4}$ were made using variable reluctance differential transducers. The transducers were installed in the testing chamber and connected to pressure taps by tubes. Among the surface quantities, the agreement between the current calculations and measurements is the poorest for pressure. This discrepancy is particularly puzzling since the agreement for both heat transfer and the locations for separation and reattachment is excellent. The trends of the two data sets are qualitatively consistent; however, the computational results are consistently higher than the measured values. The 42 percent discrepancy near the peak pressure location on the flare is very obvious; however, differences of this magnitude are also present along the hollow cylinder. In fact, this difference is a constant. If the experimental heating rate values are multiplied by a factor of 1.4, agreement between the two data set becomes very good. The source of the differences remains an open issue.

Computed Flow-Field Features. Figures 5a through $5 \mathrm{~d}$ provide an overall description of the flow-field features for the experimental flow conditions. Figure 6 provides more details for the pressure and density field in the reattachment and outer corner expansion regions. Locations for separation (S) and reattachment $(R)$ are denoted on each figure. Flow-field temperatures experience maximum values of $630 \mathrm{~K}$ (Fig. 5a) while a major portion of the shock layer remains supersonic as indicated by the Mach 1 and Mach 9.8 contours included in Fig. 5b. Also shown in Fig. 5b are particle traces or streamlines that provide an indication of the size of the separation region within which lies a single vortex.

The scalar pressure contours (ratio of local scalar pressure, nkT, to the corresponding free-stream value, where $T$ is the overall kinetic temperature ${ }^{11}$ ) presented in Fig. 5c and the density contours (Fig. 5d) provide an overall perspective of the shock strengths and the impact of the separated region. At the leading edge, a scalar pressure contour of 5 is shown; however, values greater than 10 occur near the surface. At the surface (see Fig. 3c), the maximum pressure (normal momentum) near the leading edge is about 6 times the free-stream value. The differences in the surface pressure and the scalar pressure adjacent to the surface are indicative of the nonequilibrium effects that persist downstream of the leading edge to a $x$-location of about $15 \mathrm{~mm}$. The leading edge shock produces a maximum density ratio of 2.5 near $x=22 \mathrm{~mm}$, then decreases to a value of about 1.7 prior to the shock-shock interactions. The scalar pressure rise across the leading edge shock decreases to a value of about 2.5 prior to the shockshock interactions.

The flare-induced adverse pressure gradient is evident in Fig. 5c where the iso-pressure lines coalesce into a separation shock that compresses the flow to a maximum density 14.4 times the free-stream value. An enlarged view of the density and scalar pressure contours in the reattachment region and beyond are presented in Fig. 6a and 6b, respectively. Downstream of reattachment, density ratios as large as 8 are achieved on the surface. Also, the maximum scalar 
pressure ratio of 57 occurs while the maximum surface pressure is 53 times free stream (Fig. 3c).

As mentioned previously, the length of the flare for this test case is such that the shear layer reattachment is quite close to the top of the flare and the maximum pressure downstream of reattachment is obviously being influenced by the outer corner expansion. Consequently, the calculated flare surface properties for this test case will be extremely sensitive to the size of the predicted separation region.

\section{B. Double Cone Results}

The double-cone problem selected for investigation (Fig. 7) provides stronger shock interactions than the previous problem because of the large second cone angle. This model size and configuration have been investigated previously for high enthalpy (27.8 MJ/kg) nitrogen flow where the experimental portion of the study was conducted in the T5 shock tunnel at the California Institute of Technology and the computational studies were made at the University of Minnesota. (See Refs. 8 and 9). As in the present study, these tests were at relatively low free-stream Reynolds number conditions; however, the flow conditions were substantially different from the present conditions. For example, the lowest density $\left(2.7 \times 10^{-3} \mathrm{~kg} / \mathrm{m}^{3}\right)$ case investigated with a second cone angle of $65^{\circ}$ was almost an order of magnitude larger and the free-stream velocity $(6.1 \mathrm{~km} / \mathrm{s})$ and temperature $(1208 \mathrm{~K})$ were much higher than the primary case selected for the current investigation. The combination of these flow conditions and a relatively low wall temperature $(500 \mathrm{~K})$ precluded practical DSMC simulations. Consequently, the baseline flow conditions selected for investigation are the same as those for the hollow cylinder-flare problem. In addition, the density is varied by two orders of magnitude to examine the interactions as a function of rarefaction. Density and Reynolds number values are included in Table 2; the characteristic length is the maximum model diameter.

The previous investigation at high enthalpy conditions was complicated by the fact that the chemical reactions are coupled to the fluid motion and that viscous effects are significant. Many questions arose from the initial computational investigations (Ref. 17), one of which was the source of large-amplitude steady-state variations in surface pressure and heat transfer rate. Furthermore, the data that could be extracted from these measurements were very limited.
The need for additional fundamental studies concerning the shock-shock interactions is apparent. Such an effort by Olejniczak et al. is reported in Ref. 18 where inviscid, perfect gas shock interactions on doublewedge geometries are investigated computationally to better understand the gas dynamics of the resulting shock interactions. Results from the Ref. 18 study demonstrated the existence of the large-amplitude steady-state pressure variations and the various types of interactions as a function of wedge angles and Mach number. The current investigation includes the viscous effects for hypersonic cold flow conditions for a range of Reynolds number conditions and a fixed geometry.

For the selected geometry, the shock is attached to the first-cone apex, and detached from the second cone. This geometry produces a strong shock interaction and a large separation zone, the size of which is very sensitive to the flow conditions. The flow field schematically illustrated in Fig. 8 highlights some of the features observed from previous computational and experimental investigations of high enthalpy flows. The features of the interactions are similar to those of the Edney ${ }^{19}$ Type $V$ shock interaction. As will be demonstrated by the current calculations, the flow features for the highest Reynolds number case have similarities yet differences from those shown in the schematic. As the density is reduced and the viscous effects become more significant, the shock layer and shocks merge, and the flow features depicted in Fig. 8 are no longer appropriate.

The DSMC computational domain for the baseline case $\left(\operatorname{Re}_{\infty, \mathrm{d}}=24,719\right)$ is shown in Fig. 9 with additional information concerning the grid and simulation parameters. A 7-region domain was used where the number of cells are listed for each region along with the number of subcells per cell. For this simulation, most of the cells were subdivided into $9(3 \times 3)$ subcells. The time step for region one was $5 \mathrm{~ns}$. The current DSMC simulations are not time consistent since the regions are not advancing with the same time step (the time step is constant for all cells within a given region). If the flow had any significant unsteadiness, the current DSMC results would be in error since the simulations are not time consistent and also, the macroscopic properties are time averaged quantities.

For the four remaining cases investigated, an 8-region domain was used where the eighth region was placed upstream of the cone apex. Also, four $(2 \times 2)$ subcells per cell were used. With decreasing density, 
the computational domain was adjusted so that the outer region boundaries were specified either as free stream or outflow vacuum boundaries.

As discussed earlier, all Navier-Stokes computations used a constant number of grid points $(512 \times 512)$, and the outer boundaries were adjusted to capture the flow disturbances produced by the model. Results of the Navier-Stokes calculation for the highest density condition indicated some unsteadiness, where the separation point oscillated back-and-forth. Since the Navier-Stokes calculation was not time consistent, results for this calculation are not presented.

\section{Computational Results}

Surface results from the DSMC simulations are presented in Fig. 10. A comparison with the NavierStokes results is then presented for the extent of separation (Fig. 11) and for surface distributions (Fig. 12) at the highest common Reynolds number $(8,232)$ condition. This is followed by comments on possible unsteadiness for the DSMC results at the highest Reynolds number case (Fig. 13). The subsequent discussion focuses on the flow-field features (Figs. 14 and 15) for the highest Reynolds number $(24,719)$ case and then on the influence of rarefaction on the flow field as demonstrated by the scalar pressure field results (Fig. 16) from the DSMC simulations. This section is concluded with a comparison of DSMC and Navier-Stokes results for flow-field features (Figs. 1718) for the 8,232 Reynolds number condition.

DSMC Surface Results. Figures 10a-10c present a summary of the DSMC calculated surface results for skin friction, heating, and pressure in coefficient form for each of the five flow conditions (density variations). Several trends with rarefaction are evident and consistent with what one would expect. Along the first cone and for locations not influenced by the second cone, the coefficients for friction, heating, and pressure all increase with increasing rarefaction. This is the correct trend where a larger fraction of the momentum and energy of the gas is transfered to the surface as the flow becomes more rarefied. Also, as the flow becomes more rarefied, it is more resistant to separation ${ }^{16,20}$. Information concerning the location and extent of separation are tabulated in Table 2. For the lowest density condition, the calculation indicates no separation. The location of separation (Fig. 10a) corresponds well with the experimental observation at higher density ${ }^{20}$ in that it occurs at the first inflection point in the pressure distribution. (See Fig. 10c for the location of maximum slope of the initial pressure rise.)

For the most rarefied case $\left(\operatorname{Re}_{\infty, \mathrm{d}}=247\right)$, the influence of the second cone on the first cone surface properties extends approximately to the cone apex. This was determined by comparing the surface properties of a single $25^{\circ}$ cone (wetted length $=0.1 \mathrm{~m}$ ) with that of the double cone.

Along the second cone, the locations of maximum heating, maximum pressure, and reattachment coincide. With increasing Reynolds number, the location of these quantities move aft. Also, with the exception of the highest Reynolds number case, the magnitude of the pressure coefficient increases with increasing Reynolds number. The specific reason for the change in the reattachment pressure coefficient is not clear. Two potential sources, separately or combined, could be the changes in the shock interactions and the fact that the location of reattachment has moved sufficiently close to the outer corner expansion, with increased pressure relief.

Data for the double-cone drag coefficient as a function of Reynolds number are included in Table 2. Presented are both the total drag coefficient and the drag coefficient due to pressure. Because of the strong influence of the shock interactions, the functional behavior with Reynolds number is quite different from that for a convex blunt body. For such a body, the drag coefficient increases monotonically with decreasing Reynolds number and asymptotically approaches a free molecular limit.

Comparison of DSMC and Navier-Stokes Surface Results. Figure 11 and Tables 2 and 3 present a summary of the computed data concerning separation, reattachment, and the extent of separation. With respect to the Navier-Stokes results, DSMC predicts a delayed separation and an earlier reattachment, hence, a smaller separation zone. Discrepancies at the lower Reynolds number conditions are to be expected since translational nonequilibrium effects are significant at these conditions-conditions for which the shear stress and heat flux can no longer be expressed in terms of first order gradients of other macroscopic quantities.

For the current problem, the Reynolds number at which the Navier-Stokes computations provide an accurate description is not known; however, the case for Reynolds number equal 8,232 (no evidence of unsteadiness) or somewhat higher should be 
investigated in more detail. This is particularly true since the discrepancy for the separation extent $\left(s / L_{C}\right)$ is 40 percent at this condition. For the DSMC simulation, the adequacy of the grid resolution is the basic question. Even though a number of different grids and grid adjustments were made to arrive at the grids used in this study, a more systematic grid sensitivity study would be instructive for the double-cone problem. For the Navier-Stokes computations, grid resolution is not believed to be an issue with the $512 \times 512$ grid. The fundamental question for the Navier-Stokes computations concerns the inherent shortcomings in the Navier-Stokes equations for modeling the shock wave structure and the extent to which this deficiency influences the nature of the interactions and its impact on surface quantities.

The differences in predicted separation extent are reflected in the calculated surface properties (Fig. 12). For skin friction (Fig. 12a) and heat transfer (Fig. 12b), the agreement between the two calculations is fair. Outside the separation region, the DSMC results are low with respect to the Navier-Stokes values. Since discrepancies occur upstream of the interactions, the source of the differences could be explored for a single cone problem. For surface pressure distribution (Fig. 12c), the agreement is very good when account is taken for the different locations for separation/reattachment. After the cone apex region and prior to any influence due to the second cone, both computations are in very good agreement. Also, the predicted values are slightly higher than the inviscid cone value $e^{21}$ of 0.405 .

Flow-Field Results for $\mathrm{Re}_{\infty, \mathrm{d}}=24.719$. In contrast to the Navier-Stokes calculation for this flow condition, the DSMC simulation gave no evidence of unsteadiness-although unsteadiness is difficult to detect when the extracted macroscopic properties are the result of time averaging. Certainly, no large scale unsteadiness is evident from the data (Fig. 13) for the time evolution of the separation and the reattachment locations.

Figures 14 and 15 present details concerning the flow-field structure resulting from a DSMC simulation for the $\operatorname{Re}_{\infty, \mathrm{d}}=24,719$ condition. Similarities with the schematic shown in Fig. 8 are evident with respect to the following: a transmitted oblique shock formed by the intersection of the oblique shock from the first cone and the shock from the separation zone; the intersection of the stronger oblique shock with the bow shock from the second cone; a large region of separated flow with a large vortex (Fig.14e) and underlying small vortices; and the presence of a large subsonic region outside of the separation zone. Obvious differences are the absence of an impinging supersonic jet even though a finger of supersonic flow approaches the surface (Fig. 15a). Reattachment occurs subsonically followed by a supersonic expansion about the top cylindrical extension.

For Mach 9.91 air, the pressure ratio across a normal shock is 114 . Figure $14 \mathrm{c}$ presents the calculated scalar pressure contours which include the normal shock value. Most of the region aft of the second oblique shock is at a local-to-free-stream pressure ratio of approximately 95 . Included in Fig. $14 \mathrm{c}$ is the approximate location of the dividing streamline as indicated by the curve with arrows. Compression of the flow-possibly a transmitted shock toward the surface of the second cone-is evident beneath the oblique and bow shock intersection location. This compression is evident in both the density (Fig. 14d) and pressure contours (Fig. 15b).

As the free-stream density is reduced by two orders of magnitude, the structure of the flow field and the shock interactions change dramatically as demonstrated by the scalar pressure contours shown in Fig. 16. The transmitted oblique shock resulting from the intersection of the separation shock and oblique shock from the first cone for a Reynolds number of 24,719 (Fig. 16a) is no longer evident for a Reynolds number of 8,232 (Fig. 16b). This is in contrast to the NavierStokes results for the 8,232 Reynolds number case where a distinct transmitted oblique shock is calculated (Fig. 17b).

Figures $16 \mathrm{c}$ through $16 \mathrm{e}$ demonstrate the continued thickening of the shock wave and the merger of the outer shocks and shock layer. The disturbance produced by the double-cone model extends much farther upstream of the model than the corresponding NavierStokes results (not shown) for the lower Reynolds number cases.

Comparison of DSMC and Navier-Stokes FlowField Features for a Reynolds Number of 8,232. Mach number and density contours are presented in Figs. 17 and 18 , respectively, for the 8,232 Reynolds number case. Results from both DSMC (part a) and NavierStokes (part b) calculations are presented. Similar to the largest Reynolds number condition, a supersonic finger of flow (Fig. 17) approaches the surface, and the reattachment is at subsonic conditions. For both calculations, a very small region of supersonic flow is 
evident downstream of reattachment. The general features of the two calculations are similar for both the Mach and the density contours. However, two significant differences are evident. One is the thickness of the outer shocks. The other is the presence of an oblique transmitted shock (extending from the interaction of the cone apex and separation shocks) in the Navier-Stokes (Fig. 18) calculation and the absence of this shock in the DSMC calculation. For the DSMC calculation, there appears to be a common region where the apex shock, separation shock, and second cone bow shock interact. This common region of interaction is evident from the Navier-Stokes calculation for the 2,472 Reynolds number case (not shown).

\section{Concluding Remarks}

Results of numerical simulations of Mach 10 air flow about a hollow cylinder-flare $\left(30^{\circ}\right)$ and a double cone $\left(25^{\circ}-65^{\circ}\right)$ geometry are presented for low density flows where viscous effects are significant (Reynolds numbers less than 25,000). DSMC computations are presented for both geometries, where the emphasis was to identify the level of grid resolution necessary to produce accurate results for flow conditions involving shock-shock and shock-boundary-layer interactions. For both geometries, the cold hypersonic flow investigated produced shock interactions with large regions of separated flow.

Results of the hollow cylinder-flare investigation are presented concerning grid sensitivity, comparisons with experimental surface measurements made in the ONERA R5Ch wind tunnel, and computed flow structure. The extent of the calculated separation region is very sensitive to the grid resolution used-a coarse grid results in a smaller separation region. Results for the intermediate and fine grids investigated show good to very good agreement, respectively, with the experimental measurements for the separation and reattachment locations and surface heating. For surface pressure, the agreement between calculation and measurement is poor. The calculated surface pressures along both the cylinder and flare are uniformally high with respect to the measured values by a factor of about 1.4 .

For the double-cone geometry, the primary freestream conditions were the same as those for the hollow cylinder-flare investigation. In addition, the effects of rarefaction were considered by varying the free-stream density by two orders of magnitude. In the absence of experimental data for these flow conditions, the DSMC results are compared with those computed using a
Navier-Stokes code. For the primary or highest Reynolds number $(24,719)$ condition, results from the Navier-Stokes computation indicated some unsteadiness (small oscillations of separation location), and results from this calculation are not presented. The DSMC results for this case showed no obvious unsteadiness, and the results of the calculation are presented along with the Navier-Stokes results for four lower density conditions. Qualitatively, the computed results are consistent in terms of surface quantities and flow-field structure. Quantitatively, DSMC predicts smaller values for the extent of separation, smaller values for heating and skin friction, and good agreement for pressure, excluding the separation region and regions with significant rarefaction. Also, a noticeable difference in the flow-field structure is evident for the highest common Reynolds number case $(8,232)$ in that the DSMC calculation shows a thicker shock structure which alters the shock interactions. A grid sensitivity study should be conducted for this test case, similar to that for the hollow cylinder-flare, to ensure that the DSMC results are grid resolved. The Navier-Stokes calculations were made using a $512 \times 512$ grid; consequently, grid resolution based on previous experience is not believed to be an issue for the current study. Discrepancies in calculated surface heating and skin friction could be investigated using a single cone configuration.

\section{References}

1. Délery, J. M. and Panaras, A. G., "Shock-Wave/ Boundary-Layer Interactions in High- MachNumber Flows," in AGARD-AR-319 Vol. 1, May 1996, pp 2-1 to 2-61.

2. Moss, J. N.; Dogra, V. K.; and Price, J. M., "DSMC Simulations for a Hollow Cylinder-Flare Configuration," AIAA Paper 94-2015, June 1994.

3. Moss, J. N.; Dogra, V. K.; Price, J. M.; and Hash, D. B., "Comparison of DSMC and Experimental Results for Hypersonic External Flows," AIAA Paper 95-2028, June 1995.

4. Chanetz, B., "Study of Axisymmetric Shock Wave/Boundary Layer Interaction in Hypersonic Laminar Flow," ONERA Technical Report TR No. 42/4623, February 1995.

5. Chanetz, B.; Benay, R.; Bousquet, J.-M.; Bur, R.; Oswald, J.; Pot, T.; Grasso, F.; and Moss, J., "Study of the Laminar Shock Wave/Boundary Layer 
Interaction in Hypersonic Flow: Experimental and Numerical Aspects," First Europe-US High Speed Flow Field Database Workshop, Naples, Italy, Nov. 12-14, 1997.

6. Grasso, F. and Marini, M., "Synthesis of T2-97 Hollow Cylinder Flare Problem," First Europe-US High Speed Flow Field Database Workshop Part II, Naples, Italy, Nov. 12-14, 1997. To be published.

7. Wright, M. J.; Olejniczak, J.; and Candler, G. V., "Numerical and Experimental Investigation of Double-Cone Shock Interactions," AIAA Paper 97-0063, Jan. 1997.

8. Olejniczak, J. and Candler, G. V., "Computation of Double-Cone Experiments in High Enthalpy Nitrogen," AIAA Paper 97-2549, June 1997.

9. Candler, G. V. and Olejniczak, J., "Nitrogen Dissociation Rates in Complex Hypersonic Flows," AIAA Paper 97-2500, June 1997.

10. Bird, G. A., "The G2/A3 Program System Users Manual," Version 1.8, March 1992.

11. Bird, G. A., Molecular Gas Dynamics and the Direct Simulation of Gas Flows, Clarendon Press, Oxford, 1994.

12. Wright, M. J.; Candler, G. V.; and Bose, D., "A Data-Parallel Line Relaxation Method for the Navier-Stokes Equations," AIAA Paper 97-2046, June 1997. Accepted for publication in the AIAA Journal.

13. Borgnakke, C. and Larsen, P. S., "Statistical Collision Model for Monte Carlo Simulation of Polyatomic Gas Mixture," Journal of Computational Physics, Vol. 18, No. 4, 1975, pp. $405-420$.
14. MacCormack, R. W. and Candler, G. V., "The Solution of the Navier-Stokes Equations Using Gauss-Seidel Line Relaxation," Computers and Fluids, Vol. 17, No. 1, 1989, pp. 135-150.

15. Knight, D. D. and Degrez, G., "Shock Wave Boundary Layer Interactions in High Mach Number Flows: A Critical Survey of Current CFD prediction Capabilities," in AGARD-AR-319 Vol. 2, to be published in 1998.

16. Moss, J. N.; Price, J. M.; and Chun, Ch. -H., "Hypersonic Rarefied Flow About a Com-pression Corner-DSMC Simulation and Experiment," AIAA Paper 91-1313, June 24-26, 1991.

17. Olejniczak, J. and Candler, G. V., "A Study of Experiments Sensitive to Vibration-Dissociation Coupling Models," Proceedings of the 20th International Symposium on Shock Waves, Vol. I, B. Sturtevant, J. E. Shepherd, and H. G. Hornung, Editors, World Scientic Publishing, Singapore, pp. 323-328, 1996.

18. Olejniczak, J., Wright, M. J., and Candler, G. V., "Numerical Study of Inviscid Shock Interactions on Double-Wedge Geometries," J. Fluid Mech., 1997, Vol. 352, pp. 1-25.

19. Edney, B., "Anomalous Heat Transfer and Pressure Distributions on Blunt Bodies at Hypersonic Speeds in the Presence of an Impinging Shock," Rep. 115. Flygtekniska Forsoksanstalten (The Aeronautical Research Institute of Sweden), Stockholm, 1968.

20. Needham, D. A. and Stollery, J. L., "Boundary Layer Separation in Hypersonic Flow," AIAA Paper 66-455, June 1966.

21. Equations, Tables, and Charts for Compressible Flow, Ames Research Staff, NACA TR 1135, 1953. 
Table 1. Effect of grid resolution on calculated ${ }^{a}$ extent of separation for hollow cylinder flare

\begin{tabular}{ccccc}
\hline \hline & \multicolumn{2}{c}{ Separation/Reattachment } & & \\
\cline { 2 - 3 } Number of cells & $(\mathrm{x} / \mathrm{L})_{\mathrm{S}}$ & $(\mathrm{x} / \mathrm{L})_{\mathrm{R}}$ & $\Delta(\mathrm{x} / \mathrm{L})$ & $\Delta(\mathrm{x} / \mathrm{L})_{\mathrm{DSMC}} / \Delta(\mathrm{x} / \mathrm{L})_{\operatorname{Exp}}$ \\
\hline 13,200 & 0.823 & 1.268 & 0.445 & 0.77 \\
39,400 & 0.771 & 1.312 & 0.541 & 0.93 \\
78,100 & 0.755 & 1.322 & 0.567 & 0.98 \\
Experiment $^{5}$ & $0.76 \pm 0.01$ & $1.34 \pm 0.015$ & 0.58 & - \\
\hline \hline
\end{tabular}

aDSMC results.

Table 2. DSMC results for a $25^{\circ}-65^{\circ}$ double cone ${ }^{\mathrm{a}}$

\begin{tabular}{|c|c|c|c|c|c|c|c|c|}
\hline \multirow{2}{*}{$\operatorname{Re}_{\infty, \mathrm{d}}$} & \multirow{2}{*}{$\begin{array}{c}\rho_{\infty} \\
\mathrm{kg} / \mathrm{m}^{3}\end{array}$} & \multirow{2}{*}{$C_{D}$} & \multirow{2}{*}{$\mathrm{C}_{\mathrm{D}, \mathrm{P}}$} & \multicolumn{2}{|c|}{ Separation } & \multicolumn{2}{|c|}{ Reattachment } & \multirow{2}{*}{$\Delta \mathrm{s} / \mathrm{L}_{\mathrm{C}}$} \\
\hline & & & & $\mathrm{x}, \mathrm{mm}$ & $s / L_{C}$ & $\mathrm{x}, \mathrm{mm}$ & $\mathrm{s} / \mathrm{L}_{\mathrm{C}}$ & \\
\hline 24,719 & $4.303 \times 10^{-4}$ & 1.703 & 1.684 & 21.31 & 0.470 & 59.43 & 1.668 & 1.198 \\
\hline 8,232 & $1.433 \times 10^{-4}$ & 1.626 & 1.575 & 31.16 & 0.688 & 49.54 & 1.200 & 0.512 \\
\hline 2,472 & $4.303 \times 10^{-5}$ & 1.612 & 1.525 & 35.98 & 0.794 & 47.78 & 1.117 & 0.323 \\
\hline 823 & $1.433 \times 10^{-5}$ & 1.627 & 1.489 & 45.26 & 0.995 & 46.02 & 1.033 & 0.038 \\
\hline 247 & $4.303 \times 10^{-6}$ & 1.720 & 1.494 & - & - & - & - & 0.000 \\
\hline
\end{tabular}

${ }^{a} S$ is the wetted length and $L_{C}$ is the length of each cone segment $\left(L_{C}=50 \mathrm{~mm}\right)$.

Table 3. Navier-Stokes results for a $25^{\circ}-65^{\circ}$ double cone ${ }^{a}$

\begin{tabular}{|c|c|c|c|c|c|c|}
\hline \multirow{2}{*}{$\operatorname{Re}_{\infty, \mathrm{d}}$} & \multirow{2}{*}{$\begin{array}{c}\rho_{\infty} \\
\mathrm{kg} / \mathrm{m}^{3}\end{array}$} & \multicolumn{2}{|c|}{ Separation } & \multicolumn{2}{|c|}{ Reattachment } & \multirow{2}{*}{$\Delta \mathrm{s} / \mathrm{L}_{\mathrm{C}}$} \\
\hline & & $\mathrm{x}, \mathrm{mm}$ & $\mathrm{s} / \mathrm{L}_{\mathrm{C}}$ & $\mathrm{x}, \mathrm{mm}$ & $\mathrm{s} / \mathrm{L}_{\mathrm{C}}$ & \\
\hline 8,232 & $1.433 \times 10^{-4}$ & 26.74 & 0.590 & 51.80 & 1.307 & 0.717 \\
\hline 2,472 & $4.303 \times 10^{-5}$ & 32.85 & 0.725 & 48.34 & 1.143 & 0.418 \\
\hline 823 & $1.433 \times 10^{-5}$ & 36.16 & 0.798 & 47.94 & 1.124 & 0.326 \\
\hline 247 & $4.303 \times 10^{-6}$ & 44.64 & 0.985 & 45.63 & 1.015 & 0.030 \\
\hline
\end{tabular}

${ }^{a_{S}}$ is the wetted length and $L_{C}$ is the length of each cone segment $\left(L_{C}=50 \mathrm{~mm}\right)$. 

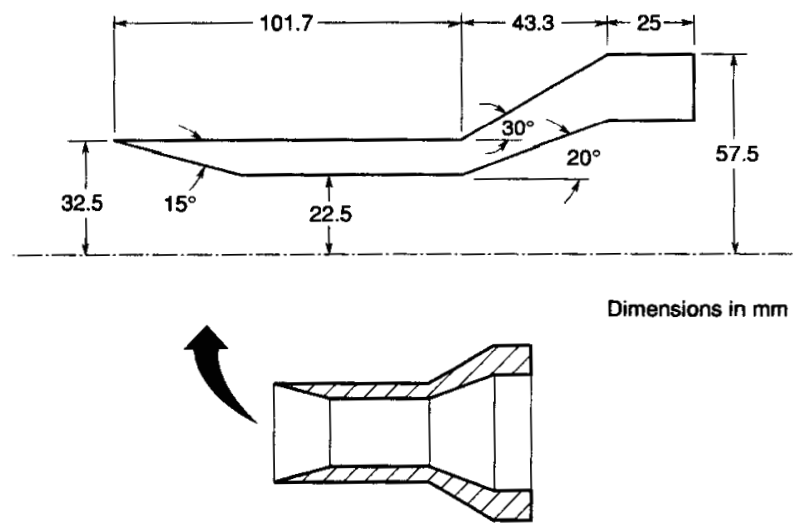

Dimensions in mm

Fig 1. Hollow cylinder-flare model.

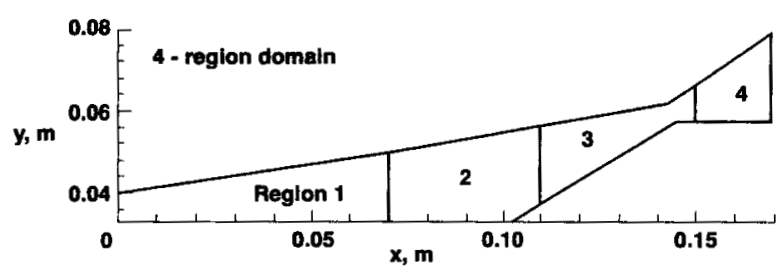

Cell ${ }^{\mathrm{a}}$ and Time Step Information

\begin{tabular}{|l|c|c|c|c|c|}
\hline Grid & Reglon 1 & Reglon 2 & Region 3 & Region 4 & $\begin{array}{l}\text { Total } \\
\text { cells }\end{array}$ \\
\hline Fine & $\begin{array}{c}70 \times 100 \\
75 \mathrm{~ns}\end{array}$ & $\begin{array}{c}100 \times 225 \\
75 \mathrm{~ns}\end{array}$ & $\begin{array}{c}120 \times 375 \\
28 \mathrm{~ns}\end{array}$ & $\begin{array}{c}20 \times 180 \\
15 \mathrm{~ns}\end{array}$ & 78,100 \\
\hline Intermediate & $\begin{array}{c}70 \times 100 \\
75 \mathrm{~ns}\end{array}$ & $\begin{array}{c}80 \times 180 \\
75 \mathrm{~ns}\end{array}$ & $\begin{array}{c}80 \times 180 \\
56 \mathrm{~ns}\end{array}$ & $\begin{array}{c}20 \times 180 \\
15 \mathrm{~ns}\end{array}$ & 39,400 \\
\hline Coarse & $\begin{array}{c}70 \times 60 \\
150 \mathrm{~ns}\end{array}$ & $\begin{array}{c}40 \times 90 \\
150 \mathrm{~ns}\end{array}$ & $\begin{array}{c}40 \times 90 \\
112 \mathrm{~ns}\end{array}$ & $\begin{array}{c}20 \times 90 \\
30 \mathrm{~ns}\end{array}$ & 13,200 \\
\hline
\end{tabular}

$a_{4}$ subcells/cell (2x2)

Fig 2. Computational domain and region information.

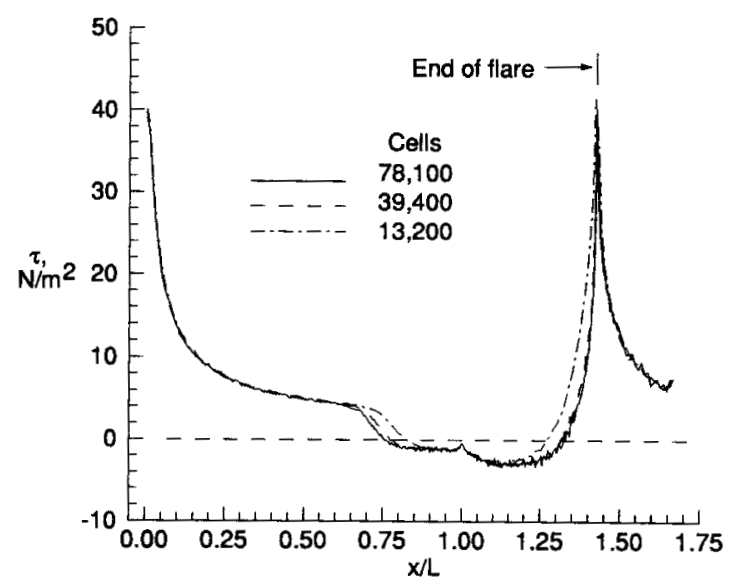

(a) Skin friction.

Fig 3. Effect of grid resolution on surface quantities.

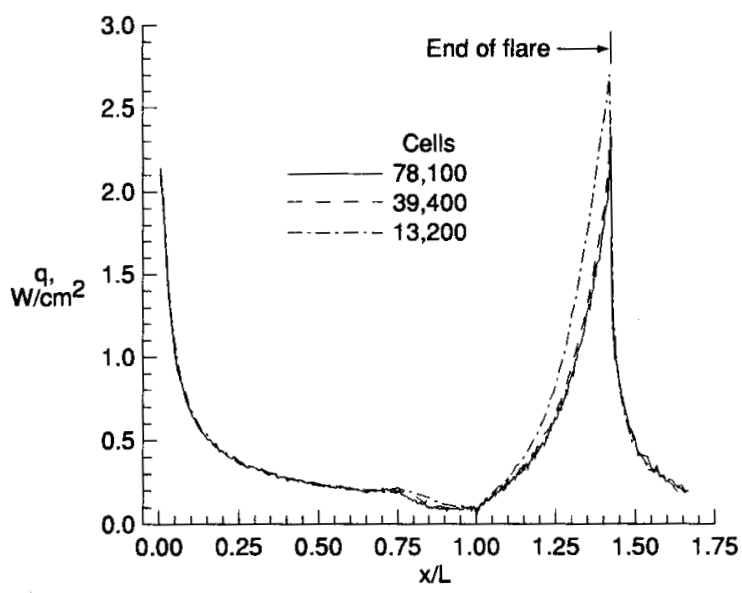

(b) Heat transfer.

Fig 3. Continued.

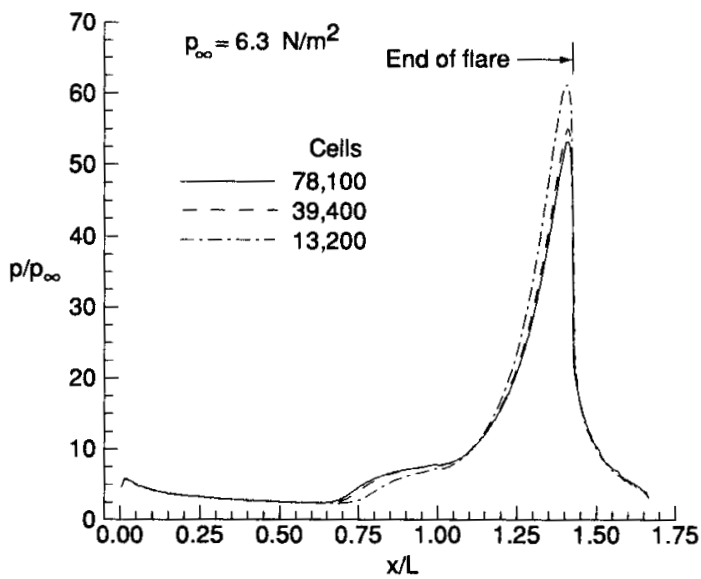

(c) Pressure.

Fig. 3. Concluded.

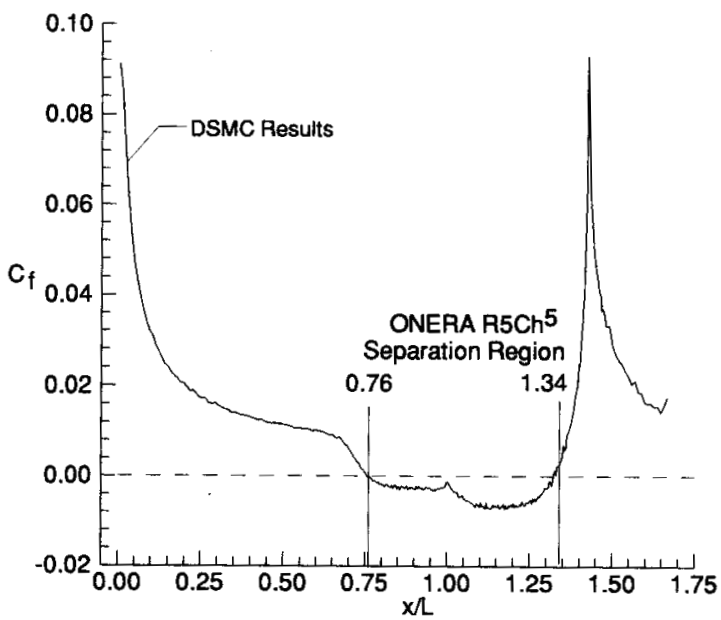

(a) Skin friction and extent of separation.

Fig 4. Comparison of calculated and measured surface results for hollow cylinder-flare model. 


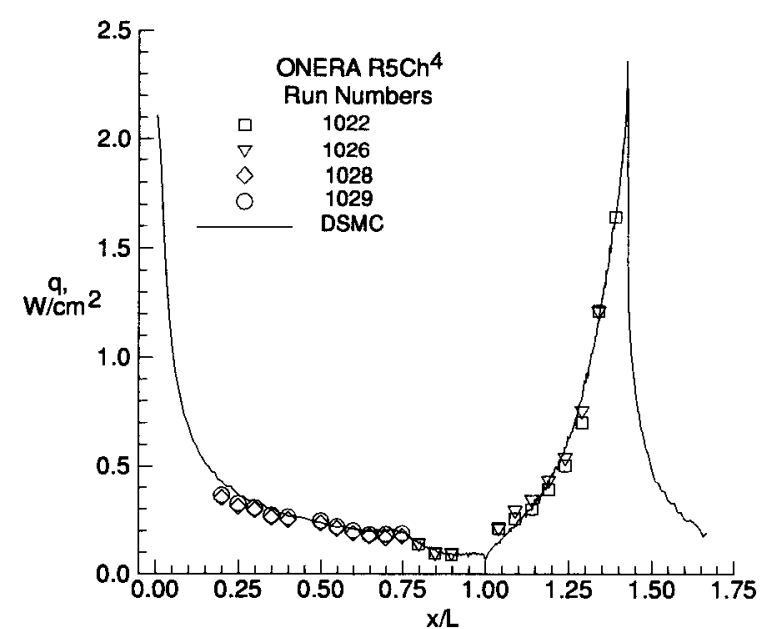

(b) Heat-transfer rate.

Fig 4. Continued.

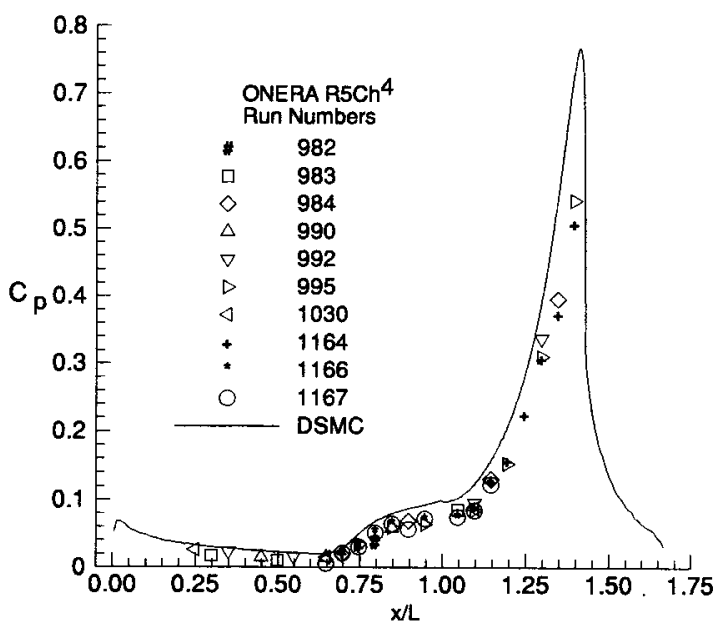

(c) Pressure coefficient

Fig 4. Concluded.

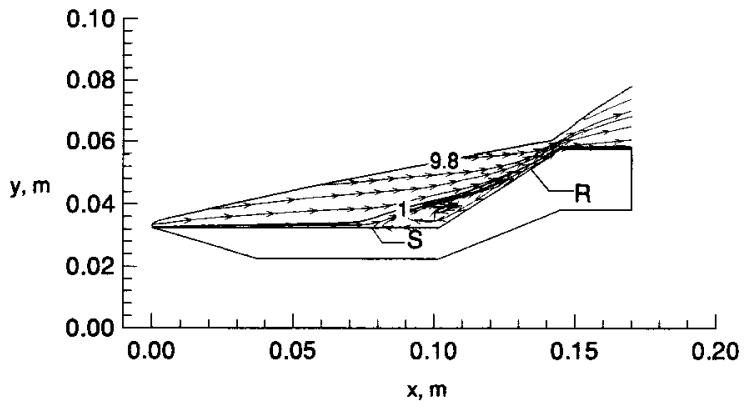

(b) Mach contours and streamlines.

Fig. 5. Continued.

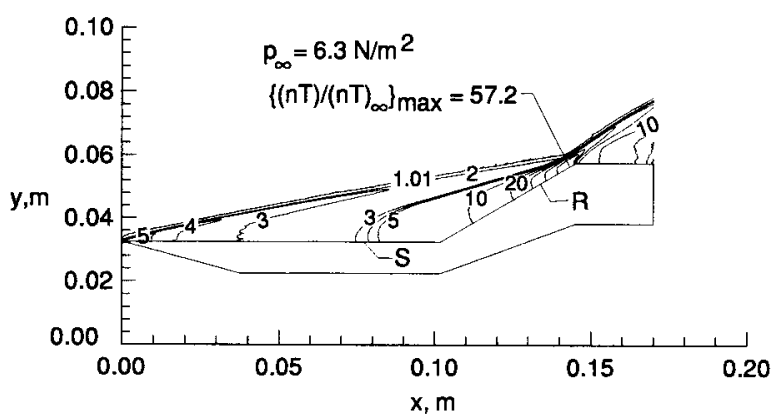

(c) Scalar pressure.

Fig. 5. Continued.

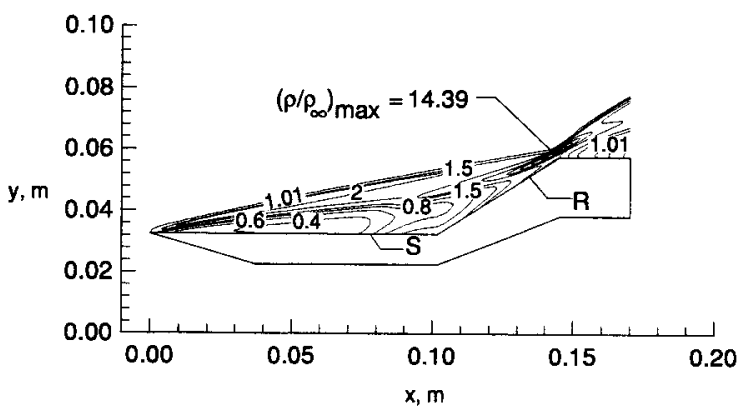

(d) Density.

Fig. 5. Concluded.

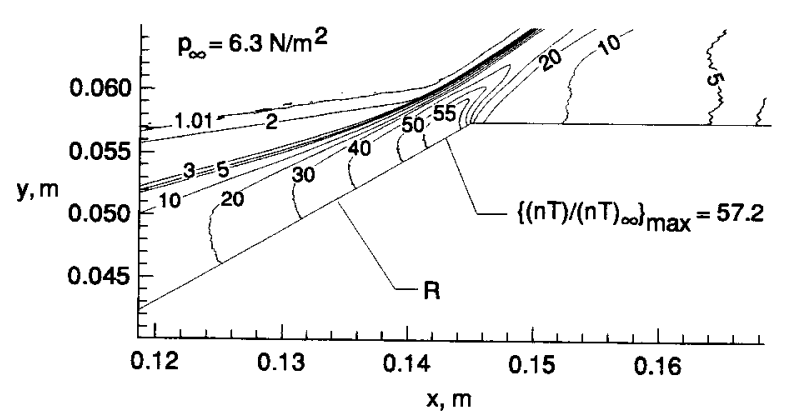

(a) Scalar pressure.

Fig. 6. Flow-field quantities in reattachment region.

Fig 5. Flow-field quantities for hollow cylinder flare. 


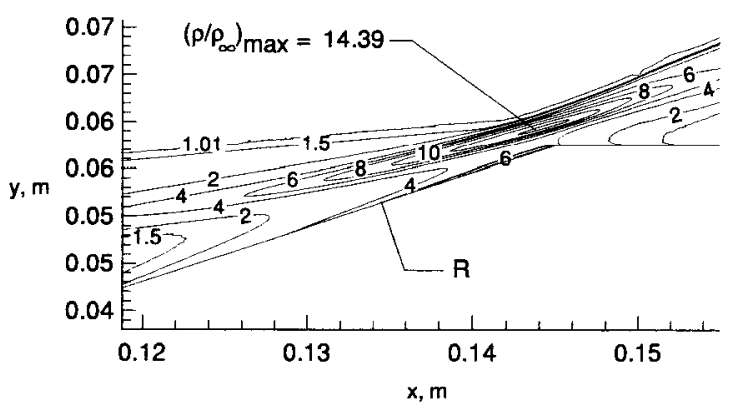

(b) Density.

Fig. 6. Concluded.

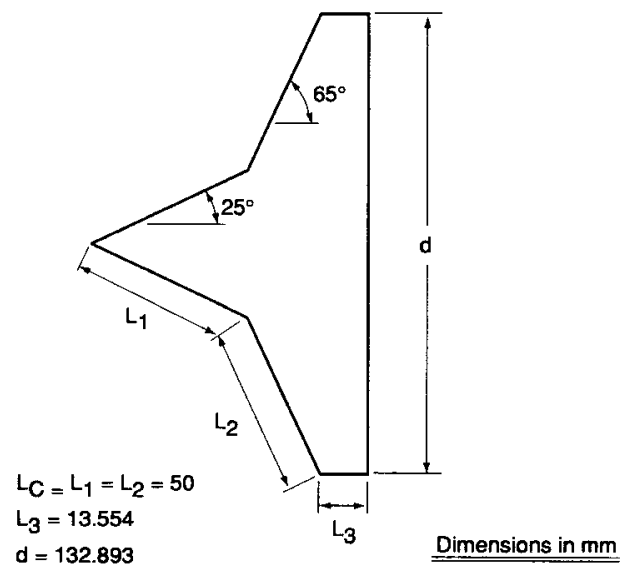

Fig. 7 Double-cone configuration.

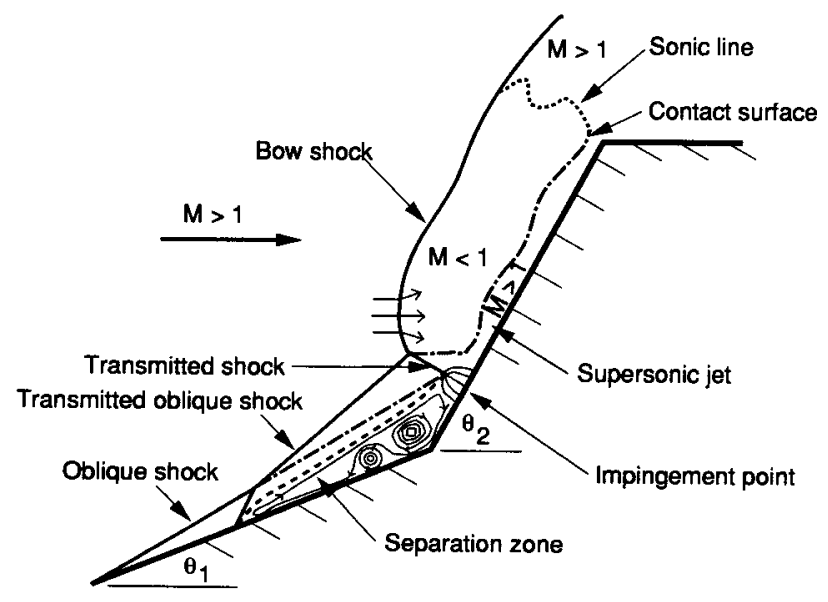

Fig. 8 Schematic diagram of viscous flow over a double cone (from Ref. 8).

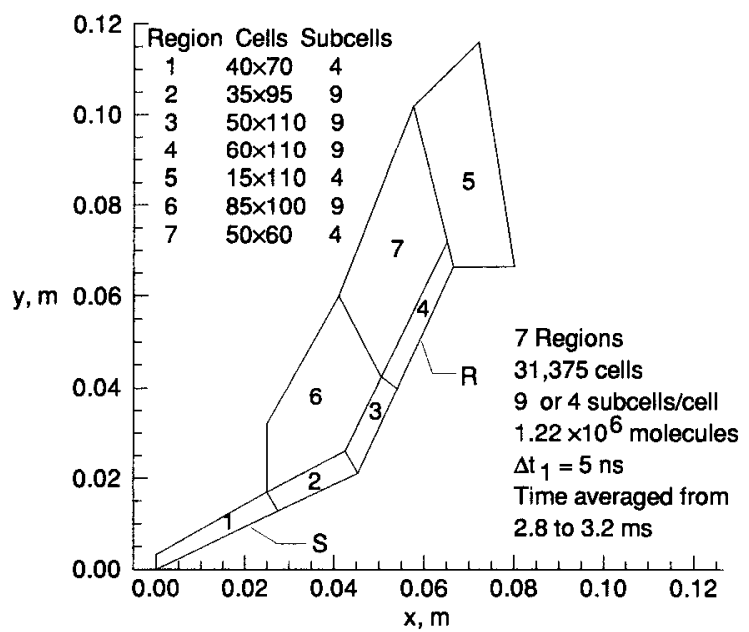

Fig. 9 Computational domain and region information for double cone at $\operatorname{Re}_{\infty, d}=24,719$.

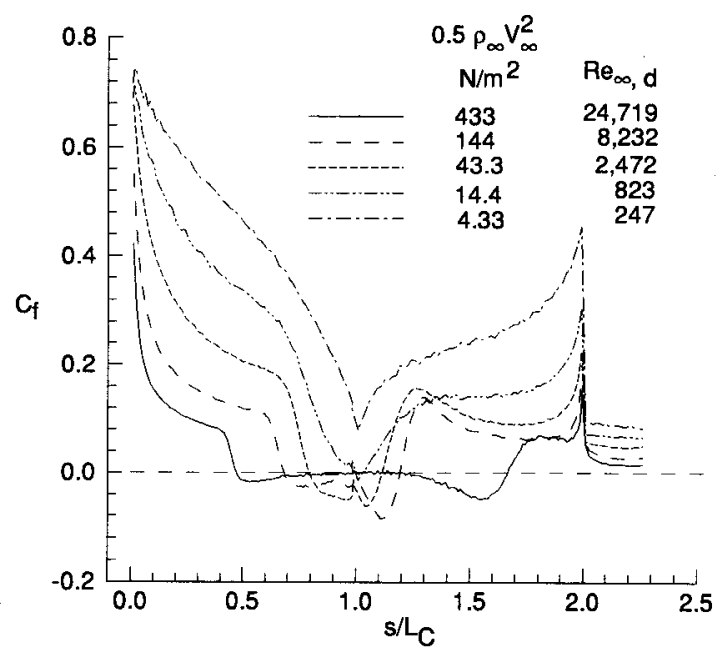

(a) Skin friction coefficient.

Fig. 10. Effect of rarefaction on surface quantities for double cone.

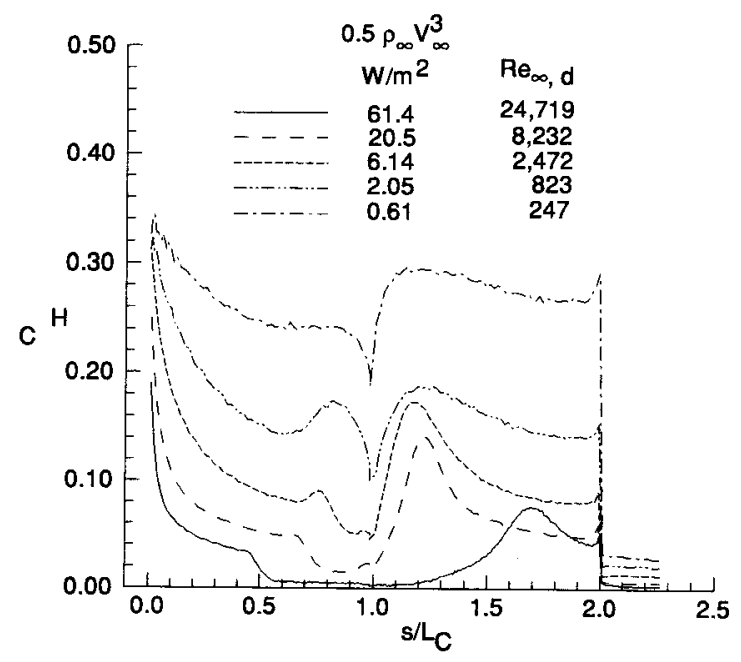

(b) Heat transfer coefficient. Fig. 10. Continued. 


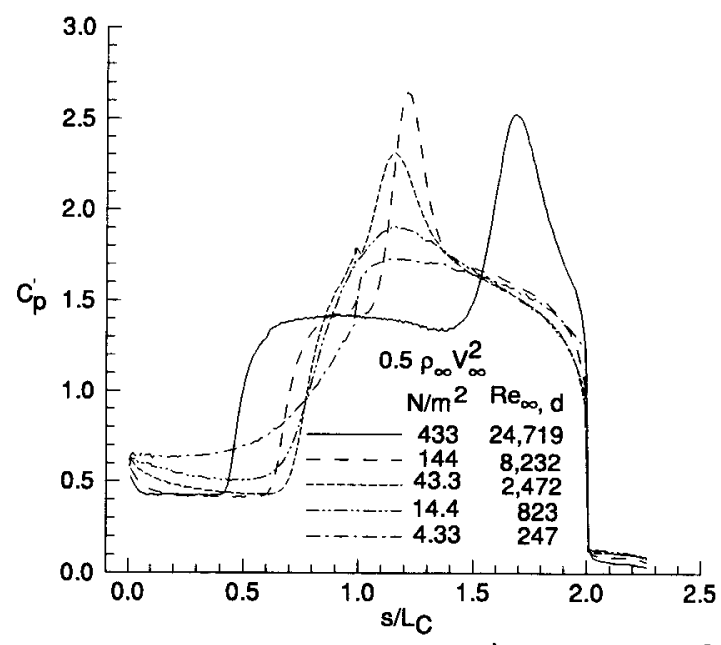

(c) Modified pressure coefficient $\left(C_{p}^{\prime}=2 p_{w} / \rho_{\infty} V_{\infty}^{2}\right)$.

Fig. 10. Concluded.

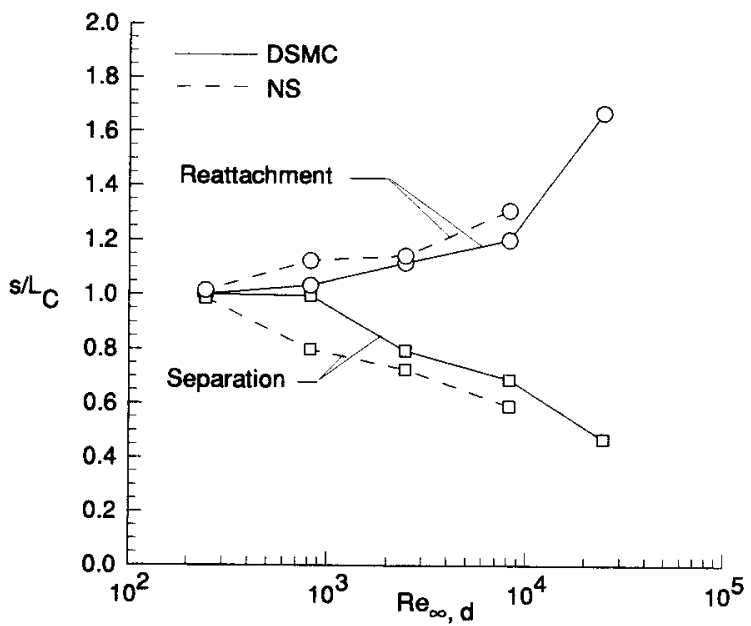

Fig. 11 Calculated extent of separation for double cone.

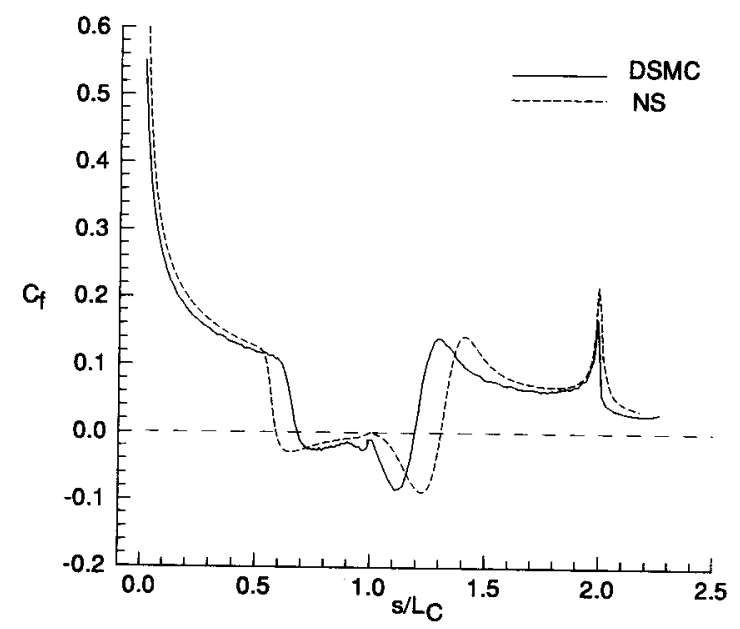

(a) Skin friction coefficient.

Fig. 12. Comparison of calculated surface results for double cone $\left(\operatorname{Re}_{\infty, d}=8,232\right)$.

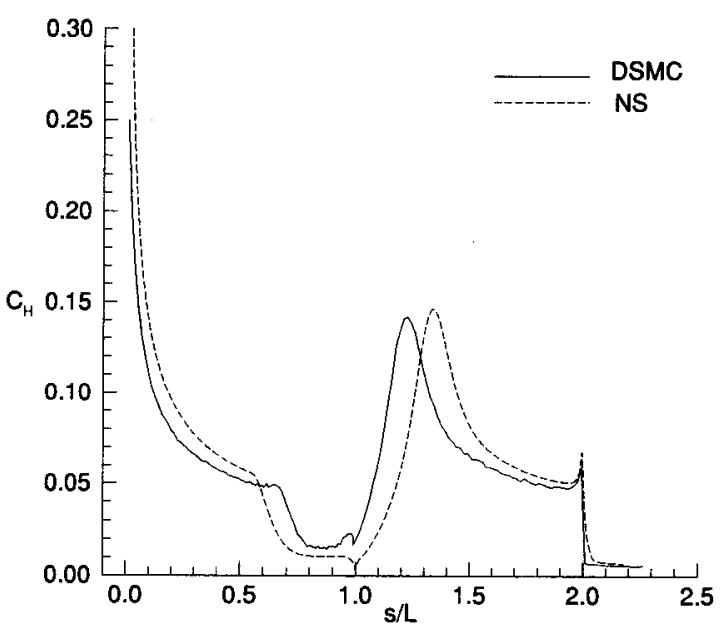

(b) Heat transfer coefficient.

Fig. 12. Continued.

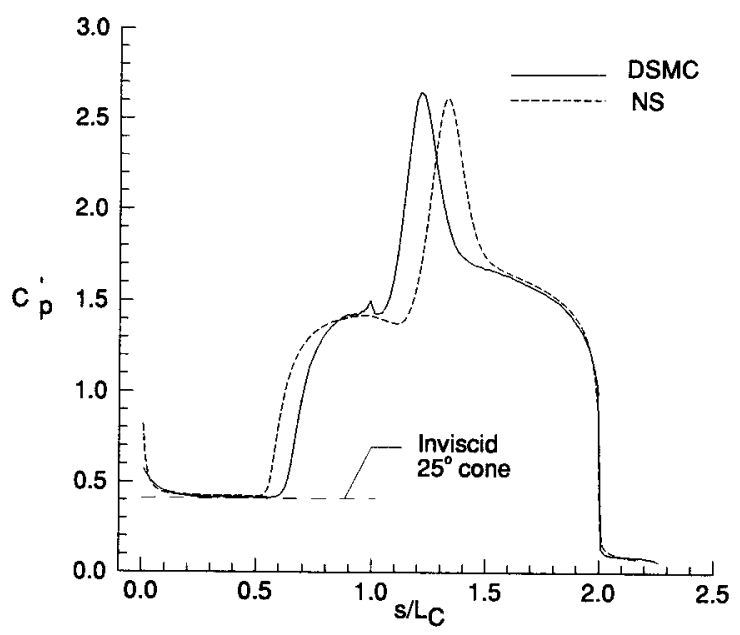

(c) Modified pressure coefficient $\left(C_{p}^{\prime}=2 p_{w} / \rho_{\infty} V_{\infty}^{2}\right)$.

Fig. 12. Concluded.

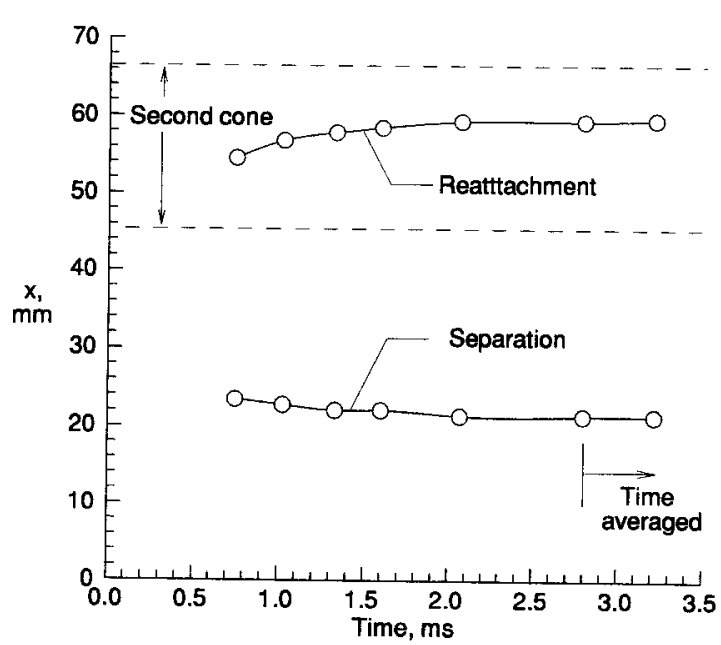

Fig. 13. DSMC simulation history for separation and reattachment locations $\left(\operatorname{Re}_{\infty, d}=24,719\right)$. 


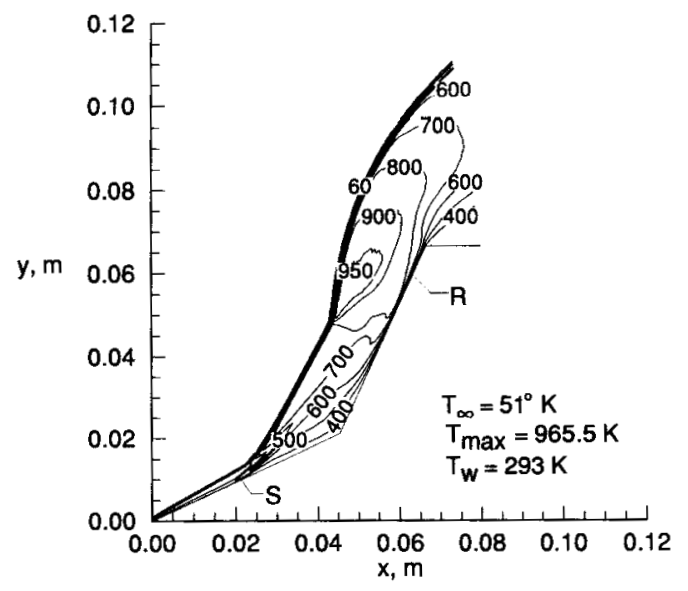

(a) Temperature.

Fig. 14. Flow-field for double cone $\left(\operatorname{Re}_{\infty}, d=24,719\right)$.

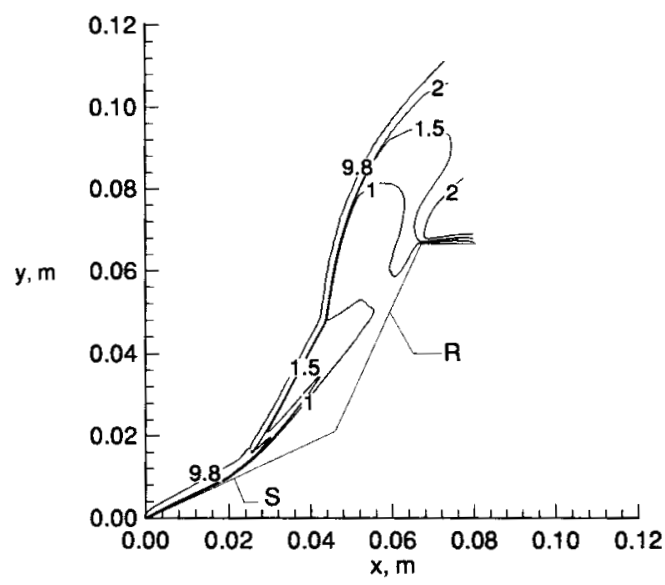

(b) Mach number.

Fig. 14. Continued.

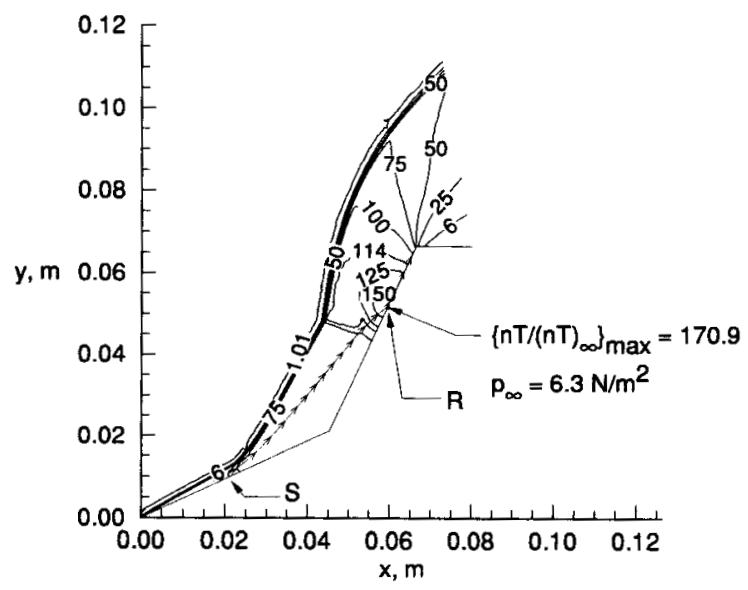

(c) Scalar pressure.

Fig. 14. Continued.

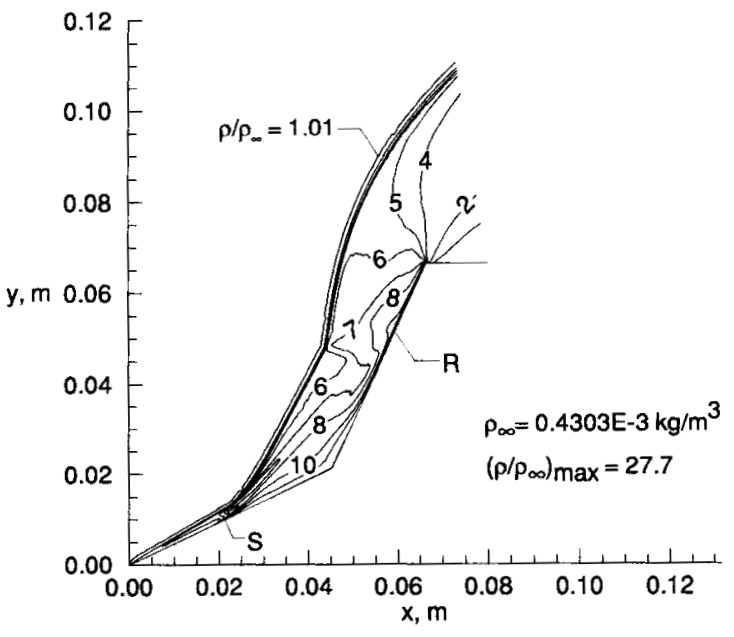

(d) Density.

Fig. 14. Continued.

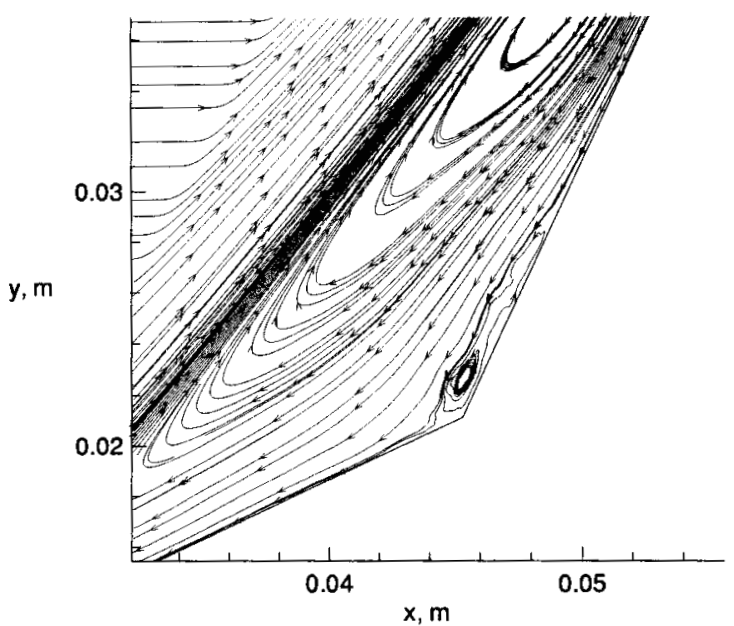

(e) Streamlines.

Fig. 14. Concluded.

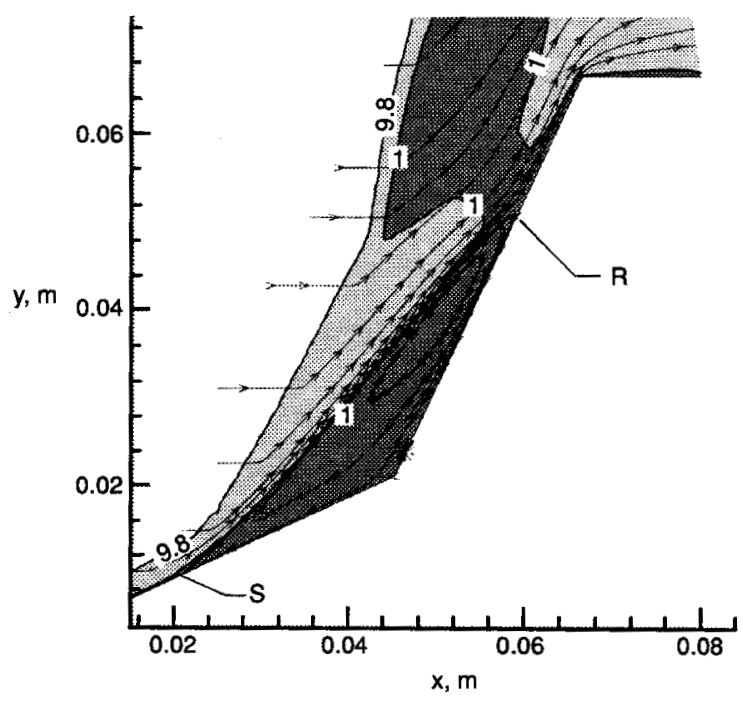

(a) Mach contours and streamlines.

Fig. 15 Flow-field quantities in reattachment region. 


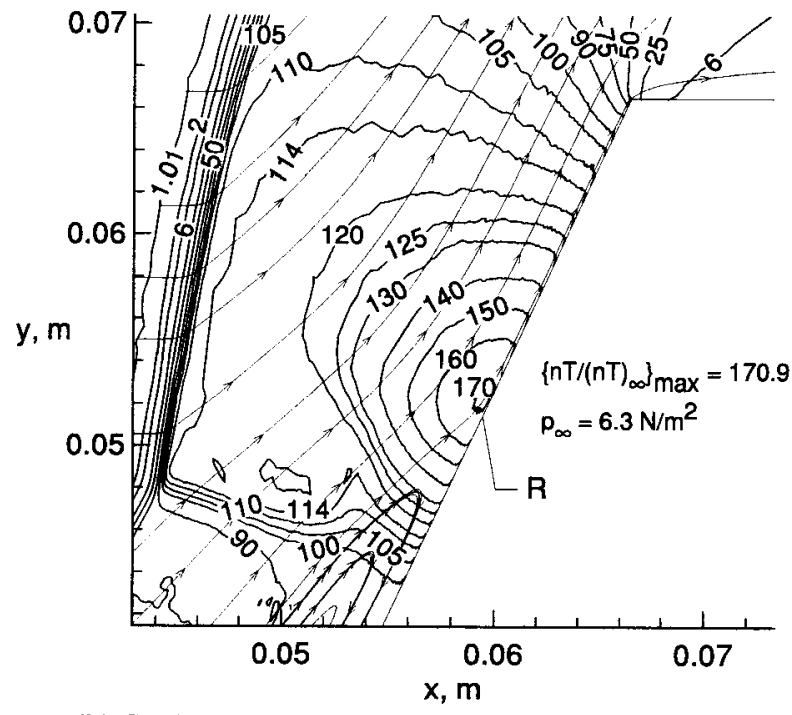

(b) Scalar pressure contours and streamlines.

Fig. 15 Concluded.

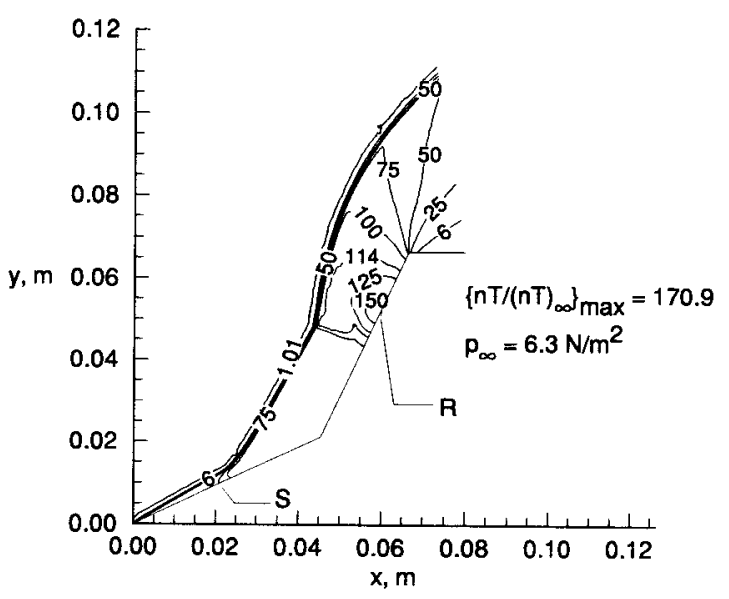

(a) $\operatorname{Re}_{\infty, d}=24,719$

Fig. 16 Effect of rarefaction on flow-field scalar pressure contours.

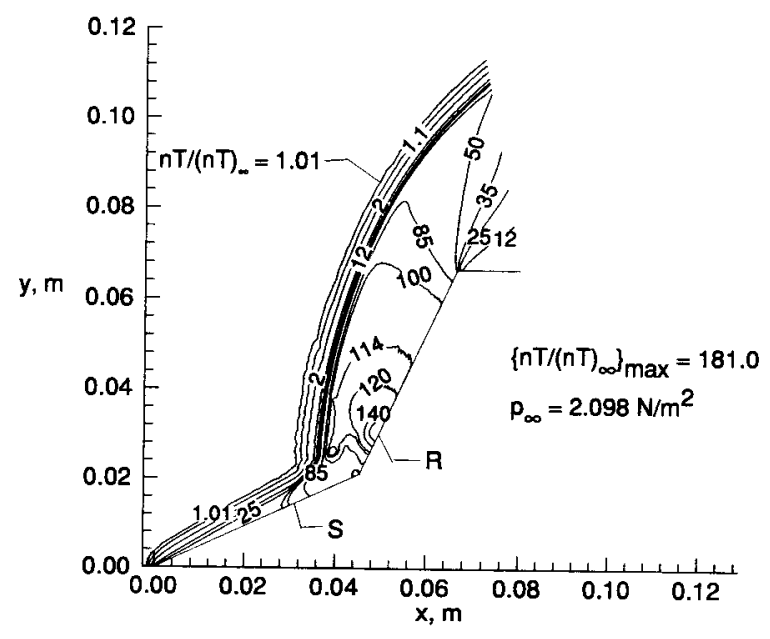

(b) $\operatorname{Re}_{\infty}, d=8,232$

Fig. 16 Continued.

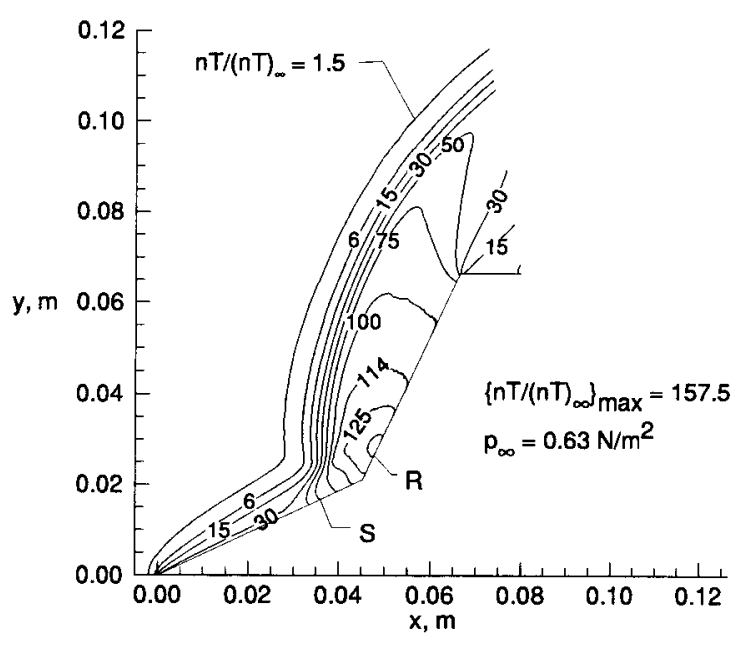

(c) $\operatorname{Re}_{\infty}, d=2,472$

Fig. 16 Continued.

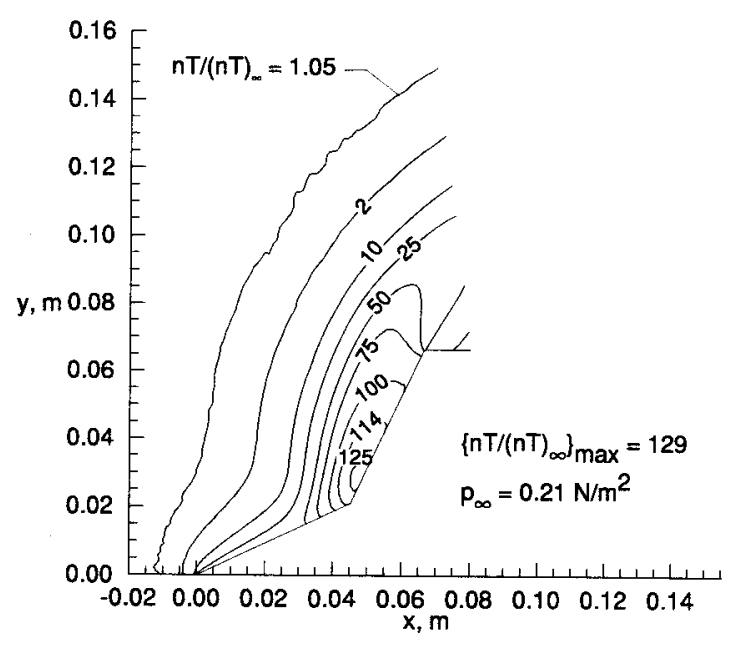

(d) $\operatorname{Re}_{\infty, d}=823$

Fig. 16 Continued.

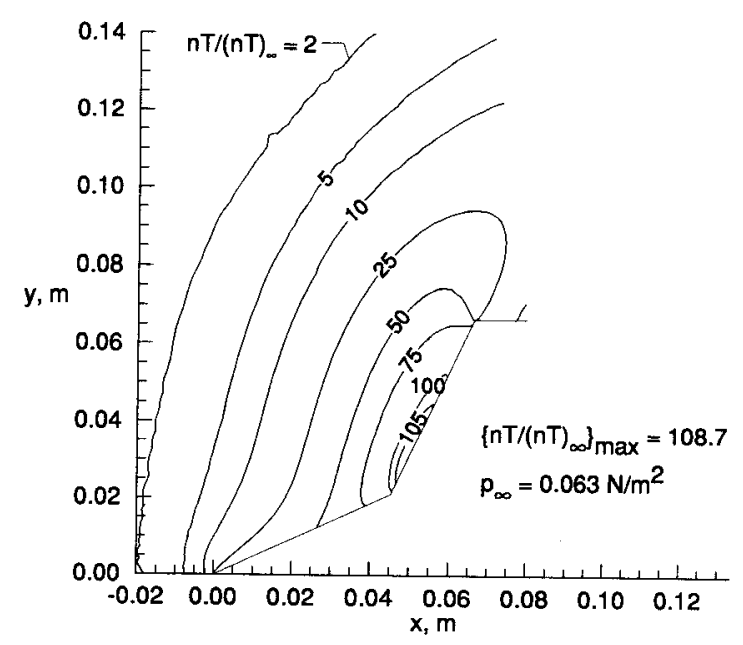

(e) $\operatorname{Re}_{\infty, d}=247$

Fig. 16 Concluded. 


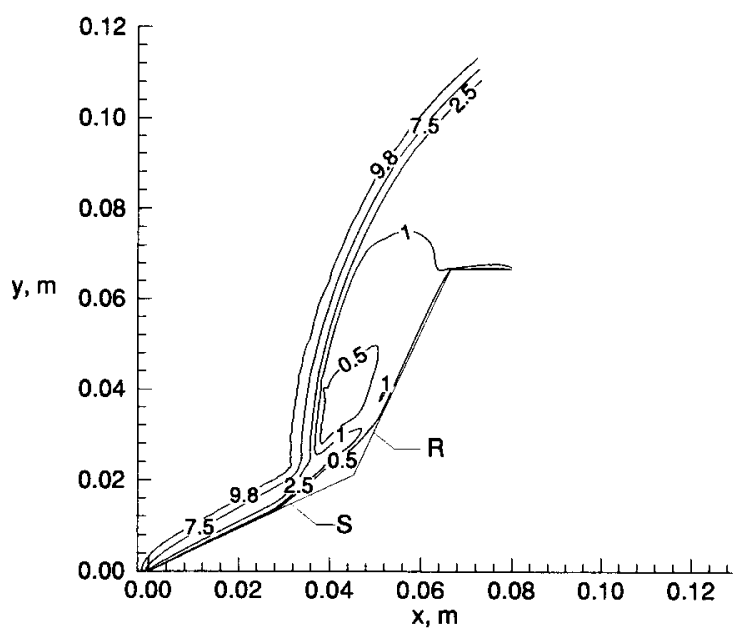

(a) DSMC results.

Fig. 17 Mach number contours for $\operatorname{Re}_{\infty, \mathrm{d}}=8,232$.

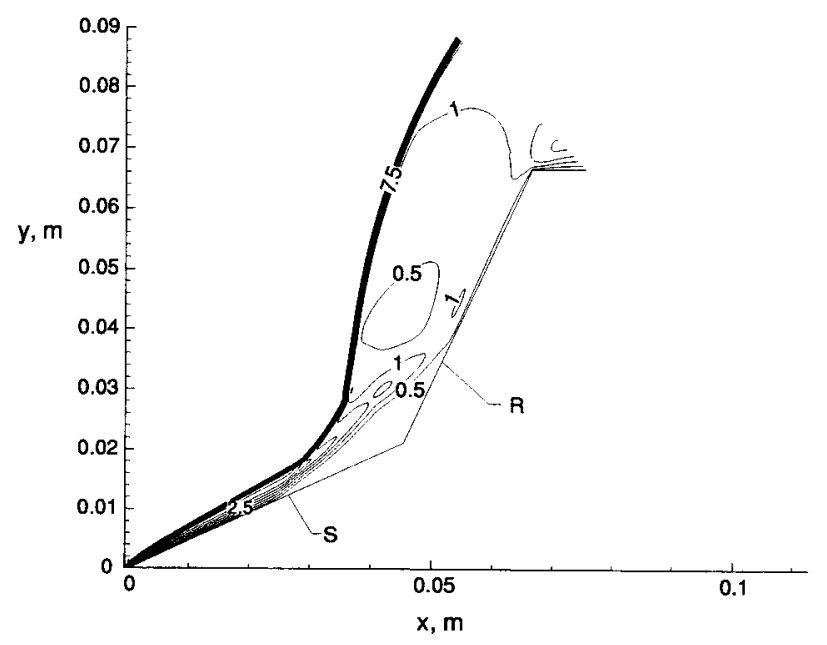

(b) Navier-Stokes results.

Fig. 17 Concluded.

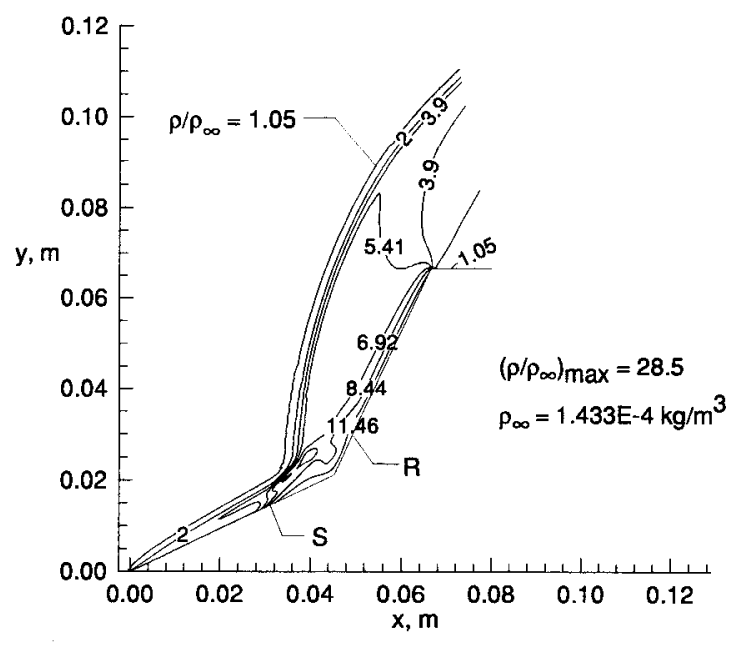

(a) DSMC results.

Fig. 18 Density contours for $\operatorname{Re}_{\infty, d}=8,232$.

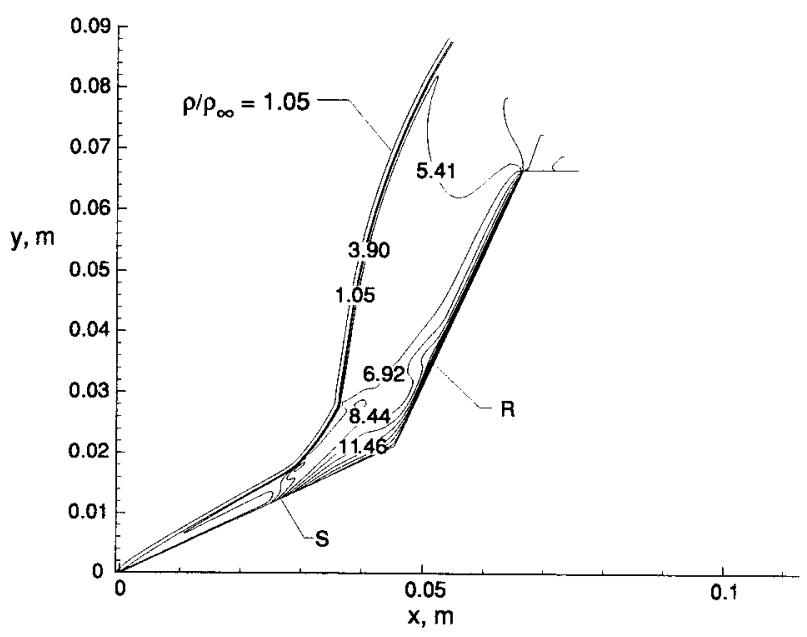

(b) Navier-Stokes results.

Fig. 18 Concluded. 